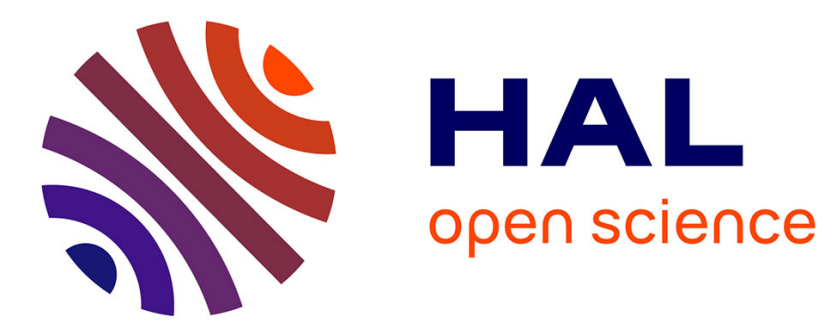

\title{
Consistency study of Lattice-Boltzmann schemes macroscopic limit
}

\author{
G. Farag, S. Zhao, G. Chiavassa, Pierre Boivin
}

\section{To cite this version:}

G. Farag, S. Zhao, G. Chiavassa, Pierre Boivin. Consistency study of Lattice-Boltzmann schemes macroscopic limit. Physics of Fluids, 2021, 33 (3), pp.037101. 10.1063/5.0039490 . hal-03160898

\section{HAL Id: hal-03160898 \\ https://hal.science/hal-03160898}

Submitted on 5 Mar 2021

HAL is a multi-disciplinary open access archive for the deposit and dissemination of scientific research documents, whether they are published or not. The documents may come from teaching and research institutions in France or abroad, or from public or private research centers.
L'archive ouverte pluridisciplinaire HAL, est destinée au dépôt et à la diffusion de documents scientifiques de niveau recherche, publiés ou non, émanant des établissements d'enseignement et de recherche français ou étrangers, des laboratoires publics ou privés. 


\title{
Consistency study of Lattice-Boltzmann schemes macroscopic limit
}

\author{
G. Farag, ${ }^{1}$ S. Zhao (赵崧), ${ }^{1, a)}$ G. Chiavassa, ${ }^{1}$ and P. Boivin $\left.{ }^{1, b}\right)$ \\ Aix Marseille Univ, CNRS, Centrale Marseille, M2P2, Marseille, \\ France
}

(Dated: January 18, 2021)

Owing to the lack of consensus about the way Chapman-Enskog should be performed, a new Taylor-Expansion of Lattice-Boltzmann models is proposed. Contrarily to the Chapman-Enskog expansion, recalled in this manuscript, the method only assumes an sufficiently small time step. Based on the Taylor expansion, the collision kernel is reinterpreted as a closure for the stress-tensor equation. Numerical coupling of LatticeBoltzmann models with other numerical schemes, also encompassed by the method, are shown to create error terms whose scalings are more complex than those obtained via Chapman-Enskog. An athermal model and two compressible models are carefully analyzed through this new scope, casting a new light on each model's consistency with the Navier-Stokes equations.

\footnotetext{
a) Also atCNES Launchers Directorate, Paris, France
}

${ }^{b)}$ Electronic mail: pierre.boivin@univ-amu.fr 


\section{INTRODUCTION}

The Navier-Stokes-Fourier (NSF) system of conservation equations is widely accepted, to study mass, momentum and energy conservation in fluid systems. Yet, its derivation from a more general and purely atomistic point of view is one of the challenges of the $6^{\text {th }}$ Hilbert problem ${ }^{1}$. Formal solutions of the Boltzmann equation $(\mathrm{BE})^{2}$ were obtained following perturbation theory ${ }^{3-5}$, but finding full thermo-hydrodynamic solutions of the BE remains an active research topic in mathematics ${ }^{6}$.

Nonetheless, this lack of theoretical understanding on the link between the NSF and BE formalisms has not slowed down the rapid development of Lattice-Boltzmann methods (LBM), now an invaluable simulation tool widely used in the engineering and scientific communities. LBM emerged in the 1980s and consist of a specific BE discretization. (i) First a discrete set of velocities is used to represent the velocity space, leading to the discrete velocity Boltzmann equation (DVBE). (ii) Second, time and space are discretized, as in most computational fluid dynamics (CFD) methods. Albeit initially limited to low-Mach athermal flows, the range of applicability has been steadily growing, to encompass compressible flows ${ }^{7-10}$, multiphase flows ${ }^{11-16}$ and combustion $^{17-19}$.

In understanding the link between the equations resolved by LBM and the macroscopic NSF system, the so-called Chapman-Enskog (CE) expansion ${ }^{20}$ is the most popular method, provided as an Appendix to most LBM papers. Yet, the CE expansion can have limitations in understanding specific aspects of modern LB methods. For instance, the aforementioned applications (beyond athermal flows) often correspond to Knudsen numbers too high for the underlying LBM theory to hold, but LBMs reportedly yields reasonable results nonetheless. The impact of the choice of collision kernel, central in the method's robustness ${ }^{21}$, is also hard to study with the CE expansion, often carried out with a simplified Bhatnagar-Gross-Krook BGK collision model ${ }^{22}$. Last but not least, the CE expansion can not be easily performed for the wide variety of models in which a LB distribution is resolved coupled to another distribution or scalar (which can represent energy, species, or any transported scalar).

The purpose of the present study is threefold. First, we provide a review of the methods traditionally used to derive the macroscopic equations from a given LBM. Second, the implicit assumptions underlying the $\mathrm{CE}$ expansion are discussed. Third, we propose a rigorous and systematic method to analyze LBMs, based on a modified equation analysis ${ }^{23-25}$ using a Taylor expansion 
in time and space. Although use of Taylor expansion to that goal is already reported in the LB literature ${ }^{26-28}$ for athermal models, the presented method is the first - to the authors knowledge - to encompass arbitrary LB numerical schemes with multi-physics coupling, arbitrary collision kernel, arbitrary force terms and arbitrary non-dimensional numbers. It will be shown that the method allows to identify error terms beyond the CE expansion, necessary to fully understand recent LB models.

The article is organized following these three goals. After a brief Section I introducing the NSF system of equations along with the necessary notations, Section II focuses on the continuous BE. We recall two popular methods used to analyze it and derive a NSF system from the BE, namely the CE expansion ${ }^{20}$ and the Grad moment system ${ }^{29}$. Section III discusses the application of the CE formalism to LBMs. In particular, we will point out the lack of consensus found in the literature around the CE expansion. Underlying assumptions and limitations are also discussed.

Section IV contains the principal novelty of the present work, following the arguments presented in Sections II-III, and proposes an alternative to the CE expansion formalism. The stepby-step algorithm to build and understand a LB scheme is thoroughly explained. Resting on a naive Taylor expansion of the numerical scheme this method is seen to be fully deductive and ansatz-free in the sense that its derivation automatically and unequivocally gives the conditions for the scheme to be consistent to an expected set of macroscopic equations in the small time-step limit $\Delta t \rightarrow 0$, while keeping the so-called acoustic scaling coefficient $\Delta t / \Delta x$ constant $^{30}$.

As a first textbook example, the classical athermal $\mathrm{BGK}^{30}$ is analyzed through the scope of the Taylor expansion in Section V. Then, in light of the proposed step-by-step algorithm, Section VI proposes a new interpretation of the LB collision kernel strictly based on macroscopic equations instead of the usual kinetic interpretation. Lastly, the scope of our new theoretical framework is illustrated for two advanced LB thermal models recently published by our group, namely the RR- $\rho^{31,32}$ and RR- $p^{7}$ models, respectfully in Sections VII and VIII.

\section{THE NAVIER-STOKES-FOURIER SYSTEM}

Before carrying out any comparison between the BE and NSF systems, it is useful to introduce the NSF governing equations, along with appropriate definitions. 


\section{A. Navier-Stokes definitions}

Mass and momentum conservation read

$$
\begin{gathered}
\frac{\partial \rho}{\partial t}+\frac{\partial \rho u_{\beta}}{\partial x_{\beta}}=\dot{m}, \\
\frac{\partial \rho u_{\alpha}}{\partial t}+\frac{\partial\left[\rho u_{\alpha} u_{\beta}+p \delta_{\alpha \beta}-\mathcal{T}_{\alpha \beta}\right]}{\partial x_{\beta}}=\rho \mathcal{F}_{\alpha},
\end{gathered}
$$

where $\rho$ is the volume mass, $u_{\alpha}$ is the local velocity vector and $p$ is the pressure. In addition, $\dot{m}$ and $\rho \mathcal{F}_{\alpha}$ are respectively any forcing term in the mass an momentum equations. These forces can model physical phenomena e.g. gravity or mass source, but they can also correspond to numerical terms such as sponge-zones ${ }^{33}$. Lastly, $\mathcal{T}_{\alpha \beta}$ is the stress tensor,

$$
\mathcal{T}_{\alpha \beta}=\mu\left(\frac{\partial u_{\alpha}}{\partial x_{\beta}}+\frac{\partial u_{\beta}}{\partial x_{\alpha}}-\delta_{\alpha \beta} \frac{2}{3} \frac{\partial u_{\gamma}}{\partial x_{\gamma}}\right)
$$

with $\mu$ the shear viscosity. The bulk viscosity is neglected in the framework of this paper, but can readily be included in the analysis.

Recombining Eqs. $(1,2)$ we obtain the kinetic tensor $\rho u_{\alpha} u_{\beta}$ equation

$$
\frac{\partial \rho u_{\alpha} u_{\beta}}{\partial t}+\frac{\partial \rho u_{\alpha} u_{\beta} u_{\gamma}}{\partial x_{\gamma}}+u_{\alpha} \frac{\partial\left[p \delta_{\gamma \beta}-\mathcal{T}_{\gamma \beta}\right]}{\partial x_{\gamma}}+u_{\beta} \frac{\partial\left[p \delta_{\alpha \gamma}-\mathcal{T}_{\alpha \gamma}\right]}{\partial x_{\gamma}}=\rho \mathcal{F}_{\alpha} u_{\beta}+\rho \mathcal{F}_{\beta} u_{\alpha}-\dot{m} u_{\alpha} u_{\beta} \text {, }
$$

not to be confused with the kinetic energy evolution equation, corresponding to half the trace of the tensor evolution Eq. (4). When the flow is assumed to be athermal, the system is fully closed by assuming, e.g.

$$
p=\rho c_{s}^{2}
$$

where $c_{s}$ is the constant sound speed.

\section{B. Fourier system definitions}

When thermal effects cannot be neglected, one needs to consider additionally the total energy density $\rho E$ equation

$$
\frac{\partial \rho E}{\partial t}+\frac{\partial\left[(\rho E+p) u_{\beta}+q_{\beta}-u_{\alpha} \mathcal{T}_{\alpha \beta}\right]}{\partial x_{\beta}}=\rho \mathcal{F}_{\gamma} u_{\gamma}+\rho \dot{q}
$$

with the total energy $E$ defined as the sum of internal and kinetic energies, $E=e+u_{\alpha} u_{\alpha} / 2$. In Eq. (6), $q_{\alpha}$ corresponds to the heat flux, and $\dot{q}$ is an energy source. 
To close the NSF system, a thermodynamic closure is required, e.g.

$$
p=\rho r T, \quad e=c_{v} T
$$

with $r=R / W, R$ the perfect gas constant, $W$ the molecular weight, $T$ the temperature and $c_{v}$ the mass heat capacity at constant volume. Laws for the heat transfer are also required, e.g.

$$
q_{\alpha}=-\lambda \partial T / \partial x_{\alpha}
$$

where $\lambda$ is the heat conductivity ${ }^{34}$. Having introduced the NSF system, let us now move forward with the BE system.

\section{CONTINUOUS BOLTZMANN EQUATION ANALYSIS}

The Boltzmann equation (BE) corresponds to the kinetic description of a gas out of equilibrium. In the absence of external forces, and assuming the gas to consist of small hard spherical particles bouncing elastically against each other, the BE reads

$$
\frac{\partial f}{\partial t}+\xi_{\alpha} \frac{\partial f}{\partial x_{\alpha}}=\Omega(f) \text {. }
$$

where $f(\boldsymbol{\xi}, \boldsymbol{x}, t)$ is the density probability function of finding particles with velocity vector $\boldsymbol{\xi}=\xi_{\alpha}$, at location $\boldsymbol{x}=x_{\alpha}$ and time $t$. The left hand-side of (9) corresponds to the free streaming of the particles, and the right hand-side takes into account their collisions. Gas kinetic theory dictate that $f$ will relax through collisions towards a Maxwellian equilibrium distribution $f^{e q}$ function ${ }^{35}$, e.g.

$$
f^{e q}=\frac{\rho}{(2 \pi R T)^{3 / 2}} e^{-\left(\|\boldsymbol{\xi}-\boldsymbol{u}\|^{2}\right) / 2 R T},
$$

for monoatomic ideal gases.

For mass, momentum and energy to be conserved through collision, they must relate to the density probability functions $f$ through ${ }^{36}$

$$
\begin{array}{r}
\rho=\int f d \boldsymbol{\xi}=\int f^{e q} d \boldsymbol{\xi}, \\
\rho u_{\alpha}=\int \xi_{\alpha} f d \boldsymbol{\xi}=\int \xi_{\alpha} f^{e q} d \boldsymbol{\xi}, \\
\rho E=\frac{1}{2} \int \xi_{\alpha} \xi_{\alpha} f d \boldsymbol{\xi}=\frac{1}{2} \int \xi_{\alpha} \xi_{\alpha} f^{e q} d \boldsymbol{\xi},
\end{array}
$$

where $\rho, u_{\alpha}$ and $E$ are the macroscopic quantities, respectively the density, velocity vector and total energy. 
For the sake of simplicity, let us consider for now the most simple collision operator, namely the Bhatnagar-Gross-Krook (BGK) collision kernel ${ }^{22}$,

$$
\Omega_{B G K}(f)=-\frac{1}{\tau}\left(f-f^{e q}\right),
$$

where $\tau$ is a characteristic relaxation time related to the viscosity of the fluid. This approximation holds reasonably when the Knudsen number $K n$, ratio of the mean free path of the particles to a characteristic flow length, is small. Note that $K n$ can also be expressed in terms of Reynolds $R e$ and Mach Ma numbers through the Von Kármán relation $K n \propto M a / R e$.

The formal derivation of the continuum NSF equations from the BE is not straightforward and usually needs a perturbation analysis which is by construction only valid for some specific asymptotic cases $^{4-6}$. For the purpose of this article, we shall recall hereafter two historical methods allowing to link the kinetic BE to the continuum NSF system.

\section{A. Hilbert and Chapman-Enskog expansions}

The question of a systematic derivation of the NSF system from kinetic theory is owed to Hilbert $^{37,38}$. Its first attempt is based on the assumption that $K n \ll 1$ and that the collision characteristic time $\tau=\epsilon \tilde{\tau}$, where $\epsilon$ is a small parameter usually identified with $K n$. The rescaled BE then reads

$$
\epsilon\left(\frac{\partial f}{\partial t}+\xi_{\alpha} \frac{\partial f}{\partial x_{\alpha}}\right)=-\frac{1}{\tilde{\tau}}\left(f-f^{e q}\right) .
$$

A singular perturbation procedure is then performed by taking the limiting case $\epsilon \rightarrow 0$. Then, we can search for the solution $f$ as an infinite expansion,

$$
f=\sum_{n=0}^{\infty} \epsilon^{n} f^{(n)}=f^{(0)}+\epsilon f^{(1)}+\epsilon^{2} f^{(2)}+\ldots,
$$

where all $f^{(n)}$ are $O(1)$. Making the ansatz that Eq. (16) is convergent we insert it inside Eq. (15). Then by assuming a scale separation between orders in $\epsilon$ and collecting terms by orders we end up with an infinite hierarchy of equations

$$
\begin{array}{r}
f^{(0)}-f^{e q}=0, \\
-\frac{1}{\tilde{\tau}} f^{(n)}=\frac{\partial f^{(n-1)}}{\partial t}+\xi_{\alpha} \frac{\partial f^{(n-1)}}{\partial x_{\alpha}},
\end{array}
$$

with $n>0$. This hierarchy of equations, called Hilbert expansion, exhibits some remarkable

properties. The first equation confirms that the zeroth order distribution $f^{(0)}$ should match the 
equilibrium distribution $f^{e q}$. The second equation shows that the $n^{\text {th }}$ equation depends on the $(n-1)^{t h}$ distribution only in a sequential manner. Then, by truncating at any order $n$ in the infinite expansion it is possible to get an approximate solution of the BE.

The problem of this solution is that it fails to capture very steep gradients where $K n=O(1)$ such as in boundary layers or shocks $3,20,39$. In those regions the distribution $f$ rapidly changes on a time scale of order $K n$ meaning that $\partial f / \partial t$ scales as $\sim f / K n$. The Hilbert expansion is then ill-equipped to deal with such applications. A way to circumvent this problem is to use the popular Chapman-Enskog expansion ${ }^{3,20}$ instead of the Hilbert expansion.

The only difference with the Hilbert expansion lies in the fact that the time derivative is now also expanded,

$$
\frac{\partial f}{\partial t}=\sum_{n=0}^{\infty} \epsilon^{n} \frac{\partial f}{\partial t_{n}}=\frac{\partial f}{\partial t_{0}}+\epsilon^{1} \frac{\partial f}{\partial t_{1}}+\epsilon^{2} \frac{\partial f}{\partial t_{2}}+\ldots
$$

where $\partial / \partial t_{n}$ denotes the contribution from the $n^{t h}$ order to the physical time derivative $\partial / \partial t$. Plugging Eqs. $(16,19)$ inside Eq. (15) leads to

$$
\epsilon\left(\sum_{n=0}^{\infty} \epsilon^{n} \frac{\partial}{\partial t_{n}}+\xi_{\alpha} \frac{\partial}{\partial x_{\alpha}}\right)\left[\sum_{m=0}^{\infty} \epsilon^{m} f^{(m)}\right]=-\frac{1}{\tilde{\tau}}\left(\left[\sum_{m=0}^{\infty} \epsilon^{m} f^{(m)}\right]-f^{e q}\right) .
$$

Assuming a scale separation between orders in $\epsilon$ and collecting terms by orders we end up with a new infinite hierarchy of equations. The leading orders in $\epsilon$ now read

$$
\begin{array}{r}
f^{(0)}-f^{e q}=0, \\
-\frac{1}{\tilde{\tau}} f^{(1)}=\frac{\partial f^{(0)}}{\partial t_{0}}+\xi_{\alpha} \frac{\partial f^{(0)}}{\partial x_{\alpha}}, \\
-\frac{1}{\tilde{\tau}} f^{(2)}=\frac{\partial f^{(1)}}{\partial t_{0}}+\frac{\partial f^{(0)}}{\partial t_{1}}+\xi_{\alpha} \frac{\partial f^{(1)}}{\partial x_{\alpha}} .
\end{array}
$$

Again, the zeroth order distribution satisfies $f^{(0)}=f^{e q}$, but a slight difference appears in higher orders, the $n^{\text {th }}$ equation now depending not only on the $(n-1)^{t h}$ but also on any $(n-m)^{t h}$ order with $m<n$. This recursive behavior means that CE expansion only addresses low-Knudsen solutions with $f$ depending only implicitly on time via the macroscopic variables appearing inside the Maxwellian Eq. (10). In other words, the CE expansion only describes solutions $f(t)$ with autonomous time dependencies $f\left(\rho(t), u(t), T(t), \nabla^{n} \rho(t), \nabla^{n} u(t), \nabla^{n} T(t)\right)$ with $\nabla^{n}$ the $n^{t h}$ order rank space derivative. More general solutions are simply out of the scope of the CE expansion ${ }^{40}$. The next step is to take successive moments of this infinite hierarchy. Integrating the first three 
order moments of Eq. (22) lead to the Euler equations

$$
\begin{aligned}
\frac{\partial \rho}{\partial t_{0}}+\frac{\partial \rho u_{\alpha}}{\partial x_{\alpha}} & =-\frac{1}{\tilde{\tau}} \int f^{(1)} d \boldsymbol{\xi}, \\
\frac{\partial \rho u_{\alpha}}{\partial t_{0}}+\frac{\partial\left[\rho u_{\alpha} u_{\beta}+\delta_{\alpha \beta} p\right]}{\partial x_{\beta}} & =-\frac{1}{\tilde{\tau}} \int \xi_{\alpha} f^{(1)} d \boldsymbol{\xi} \\
\frac{\partial \rho E}{\partial t_{0}}+\frac{\partial\left[\rho u_{\beta}(E+R T)\right]}{\partial x_{\beta}} & =-\frac{1}{\tilde{\tau}} \int \xi_{\beta} \xi_{\beta} f^{(1)} d \boldsymbol{\xi} .
\end{aligned}
$$

Similarly, the first three order moments of Eq. (23) lead to

$$
\begin{gathered}
\frac{\partial \rho}{\partial t_{1}}+\int\left(\frac{\partial}{\partial t_{0}}+\xi_{\beta} \frac{\partial}{\partial x_{\beta}}\right) f^{(1)} d \boldsymbol{\xi}=-\frac{1}{\tilde{\tau}} \int f^{(2)} d \boldsymbol{\xi} \\
\frac{\partial \rho u_{\alpha}}{\partial t_{1}}+\int\left(\frac{\partial}{\partial t_{0}}+\xi_{\beta} \frac{\partial}{\partial x_{\beta}}\right) \xi_{\alpha} f^{(1)} d \boldsymbol{\xi}=-\frac{1}{\tilde{\tau}} \int \xi_{\alpha} f^{(2)} d \boldsymbol{\xi} \\
\frac{\partial \rho E}{\partial t_{1}}+\int\left(\frac{\partial}{\partial t_{0}}+\xi_{\beta} \frac{\partial}{\partial x_{\beta}}\right) \xi_{\alpha} \xi_{\alpha} f^{(1)} d \boldsymbol{\xi}=-\frac{1}{\tilde{\tau}} \int \xi_{\beta} \xi_{\beta} f^{(2)} d \boldsymbol{\xi}
\end{gathered}
$$

which can be interpreted as a correction to the Euler equations Eqs. (24-26), leading to the NSF system of equations. Note that this system is not closed yet because we do not know how to evaluate $\int \Psi f^{(n)} d \boldsymbol{\xi}$ with $n>0$ and $\Psi=\left[1, \xi_{\alpha}, \xi_{\alpha} \xi_{\alpha}\right]$. The CE expansion being a formal search of BE solution any constraint can be used to close the system. Thus, solvability conditions are applied,

$$
\int \Psi f^{(n)} d \boldsymbol{\xi}=0, \quad n>0 .
$$

This is an essential step of the CE expansion because it allows to close the system and prevents the infinite hierarchy to impact the (low) orders of interest. Using the solvability conditions, neglecting higher orders and collecting Eqs. (24-29) the NSF system reads

$$
\begin{array}{r}
\frac{\partial \rho}{\partial t}+\frac{\partial \rho u_{\beta}}{\partial x_{\beta}}=\mathcal{O}\left(\epsilon^{2}\right), \\
\frac{\partial \rho u_{\alpha}}{\partial t}+\frac{\partial\left[\rho u_{\alpha} u_{\beta}+\delta_{\alpha \beta} p\right]}{\partial x_{\beta}}+\frac{\partial}{\partial x_{\beta}} \int \xi_{\beta} \xi_{\alpha} f^{(1)} d \xi=O\left(\epsilon^{2}\right), \\
\frac{\partial \rho E}{\partial t}+\frac{\partial\left[\rho u_{\beta}(E+R T)\right]}{\partial x_{\beta}}+\frac{\partial}{\partial x_{\beta}} \int \xi_{\beta} \xi_{\alpha} \xi_{\alpha} f^{(1)} d \xi=O\left(\epsilon^{2}\right),
\end{array}
$$

this system being closed by moments of Eq. $(22)^{30}$.

To summarize, the main CE expansion assumptions are the convergent nature of the $f$ expansion (16), the scale separation between orders in $\epsilon$ and the solvability conditions (30). 


\section{B. Grad moment system}

Aside the well known CE expansion, another attempt to link the BE to the continuum mechanics was performed by $\mathrm{Grad}^{29}$. It is of historical importance for LBMs as it introduced the use of Hermite polynomials $\mathcal{H}^{(n)}$ to analyze the BE. The main idea is to project the distribution $f$ onto the Hermite polynomials ${ }^{41}$ basis composed by $n^{\text {th }}$ rank symmetric tensors $\mathcal{H}^{(n)}$ of $n^{\text {th }}$ degree polynomials in $\boldsymbol{\xi}$, leading to

$$
\begin{aligned}
f(\boldsymbol{\xi}) & =\omega(\boldsymbol{\xi}) \sum_{n=0}^{\infty} \frac{1}{n !} \boldsymbol{a}^{(n)}: \mathcal{H}^{(n)}(\boldsymbol{\xi}), \\
\boldsymbol{a}^{(n)} & =\int f \mathcal{H}_{i}^{(n)} d \boldsymbol{\xi} \\
\omega(\boldsymbol{\xi}) & =\frac{1}{(2 \pi)^{3 / 2}} e^{-\boldsymbol{\xi} \cdot \boldsymbol{\xi} / 2} \\
\mathcal{H}^{(n)}(\boldsymbol{\xi}) & =\frac{(-1)^{n}}{\omega(\boldsymbol{\xi})} \nabla^{n} \omega(\boldsymbol{\xi}) .
\end{aligned}
$$

Assuming that $f$ is sufficiently well approximated by a $N^{\text {th }}$ order truncated version of Eq. (34),

$$
f(\boldsymbol{\xi}) \approx \omega(\boldsymbol{\xi}) \sum_{n=0}^{N} \frac{1}{n !} \boldsymbol{a}^{(n)}: \mathcal{H}^{(n)}(\boldsymbol{\xi}),
$$

it is possible to get a closed system of equations by injecting Eq. (38) into the BE (9). Taking moments of this system and using the orthogonality properties ${ }^{30,41,42}$ of the Hermite polynomials lead to a closed set of macroscopic equations, the most famous one being the Grad-13 system of equations $^{29,39}$ that describes the evolution of 13 different moments $\left(\Pi^{(0)}, \Pi_{\alpha}^{(1)}, \Pi_{\alpha \beta}^{(2)}\right.$ and $\left.\Pi_{\gamma \gamma \alpha}^{(3)}\right)$. Because of the completeness of the Hermite basis, when $N \rightarrow \infty$ the Grad moment method explores every possible BE solutions.

\section{The infinite hierarchy of equations and the closure problem}

By performing a CE or a Grad moment analysis we are trying to reduce the infinite cascade of moments to a finite system. In this process we are purposely losing some information and we expect that this ansatz was relevant for the considered physical phenomena. In the context of CFD this usually means that we are trying to extract low-Knudsen solutions out of the discretized LB model.

Whether we use the CE or the Grad moment expansion, both methods rely on a truncated expansion. To the best of the authors' knowledge the range of validity of such finite expansions 
is usually not questioned nor recalled in the LB community.

For instance, the CE expansion first assumes that the time derivative Eq. (19) and populations $f$ Eq. (16) can be expanded, but none of these assumptions seems to be explicable, particularly when we also remark that this leads to a singular perturbation analysis, meaning that the smallness parameter $\epsilon$ appears in front of the highest order derivative in the equation. Such singular perturbation expansion is known to exhibit unphysical behaviors when truncated ${ }^{43}$, i.e. the CE expansion is asymptotic rather than convergent ${ }^{30,39,40,44,45}$. Therefore, for a given physical application there is an optimal number of terms to keep in the infinite CE expansion because higherorder terms may introduce unphysical behaviors. For example, higher order approximations than NSF, namely the Burnett and Super-Burnett equations, can be derived from a truncated CE expansion of respectively the Grad-13 and Grad-26 systems ${ }^{44,46}$ but also directly from the BE itself. However, negative viscosity for high gradients and short wave instabilities of the Burnett and Super-Burnett equations ${ }^{5}$ are reported in the literature. This phenomenon was first observed by Bobylev $^{47,48}$. This means that higher order approximations in the CE expansion may lead to less stable and less physical results ${ }^{5,46}$, endorsing a non convergence of clipped infinite expansions such as the CE expansion.

However, when trying to link LBMs with some macroscopic equations we usually resort to a truncated CE expansion. This link is obtained with strong assumptions whose practical use can be reasonably questioned by the existence of Bobylev instabilities for Burnett and Super-Burnett models.

It can also be highlighted that the CE expansion is often performed with a very simple BGK collision operator, which is now hardly used in practical applications for its behavior in shear flows ${ }^{49}$ and porous flows ${ }^{50}$. Although the formal CE expansion is possible with the quadratic Boltzmann collision operator ${ }^{3,20}$, extension to complex collision kernels such as Regularized $7,31,32$ or Entropic ${ }^{51-53}$ remains unknown, at least to the authors' knowledge. In those kernels at least a part of the non-equilibrium population is systematically filtered out and replaced by a reconstructed population. This is therefore out of the scope of the formal CE expansion because finding an explicit solution $f^{(n)}$ as a function of $f^{(n-1)}, f^{(n-2)}, \ldots$ as simply as in Eqs. (21-23) would require to invert a complex collision kernel.

We have now presented the basis of the two most common analysis tools applied to the BE. Let us now focus exclusively on Lattice-Boltzmann methods, and leave the continuous BE. 


\section{LATTICE-BOLTZMANN METHODS}

\section{A. From Boltzmann to Lattice-Boltzmann}

Besides the time and space discretization on cartesian grids, the main difference between the $\mathrm{BE}$ and LBMs is the velocity space discretization.

Assume a $d$ dimensional lattice with $q$ discrete velocities $c_{i \alpha}$, referred to as the $D d Q q$ lattice ${ }^{30}$. Through discretization, Eqs. (11-13) become

$$
\begin{aligned}
\rho & =\sum_{i=1}^{q} f_{i}=\sum_{i=1}^{q} f_{i}^{e q}, \\
\rho u_{\alpha} & =\sum_{i=1}^{q} c_{i \alpha} f_{i}=\sum_{i=1}^{q} c_{i \alpha} f_{i}^{e q}, \\
\rho E & =\frac{1}{2} \sum_{i=1}^{q} c_{i \alpha} c_{i \alpha} f_{i}=\frac{1}{2} \sum_{i=1}^{q} c_{i \alpha} c_{i \alpha} f_{i}^{e q},
\end{aligned}
$$

where $f$ and $f^{e q}$ are the discretized probability density functions corresponding to their BE counterpart from Eqs. $(9,10)$. Accordingly the BE is also discretized such that we should now solve $q$ $\mathrm{BEs}$, one for each discrete velocities $c_{i \alpha}$,

$$
\frac{\partial f_{i}}{\partial t}+c_{i \alpha} \frac{\partial f_{i}}{\partial x_{\alpha}}=-\frac{1}{\tau}\left(f_{i}-f_{i}^{e q}\right)
$$

The last piece of the puzzle is to project the distributions onto the Hermite basis using Eq. (38). Because of the boundedness of the lattice a natural clip appears inside the Hermite basis. Each $D d Q q$ lattice only being able to represent $q$ independent Hermite polynomials, any Hermite polynomial beyond is in the span of the base.

On the bright side, this implies that Eq. (38) no longer involves an infinite hierarchy of equations, so it is enough to search for the solution as a finite expansion.

\section{B. The Chapman-Enskog expansion in LBM}

In a context of growing interest on LBMs, new models are being published on a monthly basis, usually including a CE expansion appendix. That CE expansion is usually left unquestioned, yet there is little consensus on its application to LBM.

To highlight this lack of consensus, we included in Tab. I a summary of different CE expansions found in four reference LBM textbooks ${ }^{30,39,54,55}$. Table I shows that only ${ }^{54,55}$ agree with 
Table I: Chapman-Enskog expansions in the lattice-Boltzmann literature.

\begin{tabular}{|c|c|c|c|}
\hline Reference textbook & $f=\ldots$ & $\partial / \partial t=\ldots$ & $\partial / \partial x=\ldots$ \\
\hline Mohamad $^{54}$ & $\sum_{n=0}^{\infty} \epsilon^{n} f^{(n)}$ & $\epsilon \frac{\partial}{\partial t_{1}}+\epsilon^{2} \frac{\partial}{\partial t_{2}}$ & $\epsilon \frac{\partial}{\partial x_{1}}$ \\
\hline Guo and Shu 55 & $\sum_{n=0}^{\infty} \epsilon^{n} f^{(n)}$ & $\epsilon \frac{\partial}{\partial t_{1}}+\epsilon^{2} \frac{\partial}{\partial t_{2}}$ & $\epsilon \frac{\partial}{\partial x_{1}}$ \\
\hline Krüger et al. ${ }^{30}$ & $\sum_{n=0}^{\infty} \epsilon^{n} f^{(n)}$ & $\sum_{n=1}^{\infty} \epsilon^{n} \frac{\partial}{\partial t_{n}}$ & $\epsilon \frac{\partial}{\partial x_{1}}$ \\
\hline Succi ${ }^{39}$ & $\sum_{n=0}^{\infty} \epsilon^{n} f^{(n)}$ & $\sum_{n=1}^{\infty} \epsilon^{n} \frac{\partial}{\partial t_{n}}$ & $\sum_{n=1}^{\infty} \epsilon^{n} \frac{\partial}{\partial x_{n}}$ \\
\hline
\end{tabular}

each other. They chose to expand the time derivative not as an infinite expansion but as a linear composition of a fast convective time $t_{1}$ and a slow diffusive time $t_{2}$, in contrast to ${ }^{30,39}$ that considers - as the historical CE - $\partial / \partial t_{n}$ as mathematical derivatives. Under the assumption that each physical phenomenon takes place on the same scale, space derivative is almost never expanded, except by ${ }^{39}$.

Despite these differences, and reassuringly, these expansions all lead to the same lowest orders corresponding to the expected Navier-Stokes equations. In analyzing error terms in the higher orders, however, these differences will lead to different results.

\section{TAYLOR EXPANSION OF A GENERIC LATTICE-BOLTZMANN SCHEME}

LBMs being extensively used by engineers and researchers as a thermo-hydrodynamic solver, it can be interesting to analyze it purely as a CFD method for macroscopic equations. In other words, knowing a given LB scheme we could simply expand it as a Taylor series in small parameter $\Delta t \rightarrow 0$. Then, we recast the scheme whose unknowns are $f_{i}$ into $q$ schemes whose unknowns are macroscopic variables such as mass, momentum and stress tensor. Lastly, we can a priori deduce in terms of nondimensional numbers consistency conditions of this scheme. This naive formalism allows to bypass the $\mathrm{CE}$ expansion assumptions, now only needing $\Delta t$ to be small, as is usual for consistency studies. This strategy will also allow to simultaneously tackle possible numerical couplings between LBM and other schemes. 
Table II: Lattice-Boltzmann notations

\begin{tabular}{llc}
\hline \hline Notation & Representation & Equation \\
\hline$f_{i}$ & Total population & $f_{i}^{e q}+f_{i}^{n e q}$ \\
$f_{i}^{e q}$ & Equilibrium population & 48 \\
$f_{i}^{n e q}$ & Non-equilibrium population & 49 \\
$\bar{f}, \bar{f}^{n e q}$ & Modified populations for $2^{\text {nd }}$ order accuracy & 50,53 \\
$f_{i}^{c o l}$ & Population after collision & 54 \\
$F_{i}$ & Forcing term & 52 \\
$\mathcal{H}^{(k)}$ & Hermite polynomials & 43 \\
$a_{\alpha_{1} \ldots \alpha_{n}}^{f,(n)}$ & Hermite moments & 45 \\
$\Pi_{\alpha_{1} \ldots \alpha_{n}}^{f,(n)}$ & Raw moments & 46 \\
$D_{\alpha_{1} \ldots \alpha_{n}}^{f,(n)}$ & Lattice isotropy defect & 47 \\
\hline \hline
\end{tabular}

\section{A. Lattice-Boltzmann definitions}

To facilitate the reading, let us now introduce LB specific quantities. For future reference, all quantities introduced in this Section are summarized in Table II.

The first Hermite polynomials read

$$
\begin{gathered}
\mathcal{H}_{i}^{(0)}=1, \quad \mathcal{H}_{i \alpha}^{(1)}=c_{i \alpha}, \quad \mathcal{H}_{i \alpha \beta}^{(2)}=c_{i \alpha} c_{i \beta}-c_{s}^{2} \delta_{\alpha \beta}, \\
\mathcal{H}_{i \alpha \beta \gamma}^{(3)}=c_{i \alpha} c_{i \beta} c_{i \gamma}-c_{s}^{2}\left[c_{i \alpha} \delta_{\beta \gamma}+c_{i \beta} \delta_{\gamma \alpha}+c_{i \gamma} \delta_{\alpha \beta}\right]
\end{gathered}
$$

where the lattice sound speed is $c_{s}=\Delta x /(\sqrt{3} \Delta t)$ for standard lattices. Higher order polynomials do not generally belong to the Hermite base of standard lattices and are not provided here $\mathrm{e}^{30}$.

For a given Hermite basis and an arbitrary population $f$ we define its Hermite moments $a_{\alpha_{1} \ldots \alpha_{n}}^{f,(n)}$ and macroscopic (raw) moments $\prod_{\alpha_{1} \ldots \alpha_{n}}^{f,(n)}$ as

$$
\begin{aligned}
a_{\alpha_{1} \ldots \alpha_{n}}^{f,(n)} & =\sum_{i}^{q} \mathcal{H}_{i \alpha_{1} \ldots \alpha_{n}}^{(n)} f_{i}, \\
\Pi_{\alpha_{1} \ldots \alpha_{n}}^{f,(n)} & =\sum_{i}^{q} c_{i \alpha_{1} \ldots c_{i \alpha_{n}}} f_{i} .
\end{aligned}
$$

Since the number of discrete velocities is finite, there always exists an order involving a non-zero 
Table III: Third-order isotropy defects of standard lattices.

\begin{tabular}{llll}
\hline \hline & $D_{x x x}^{f^{e q},(3)}$ & $D_{x x y}^{f^{e q},(3)}$ & $D_{x y z}^{f^{e q},(3)}$ \\
\hline D2Q9 & $\rho u_{x}^{3}$ & 0 & N/A \\
D3Q19 & $\rho u_{x}^{3}$ & $\rho u_{y} u_{z}^{2} / 2$ & $\rho u_{x} u_{y} u_{z}$ \\
D3Q27 & $\rho u_{x}^{3}$ & 0 & 0 \\
\hline \hline
\end{tabular}

isotropy defect $D_{\alpha_{1} \ldots \alpha_{n}}^{(n)}$ between continuous and discrete moments,

$$
D_{\alpha_{1} \ldots \alpha_{n}}^{f,(n)}=\int c_{\alpha_{1} \ldots c_{\alpha_{n}}} f d c-\Pi_{\alpha_{1} \ldots \alpha_{n}}^{f,(n)}
$$

For nearest-neighbors lattices, this isotropy defect appears from the third order $(n=3)^{30}$. For future reference, the term is provided in Table III for the athermal LB model detailed in the next Section.

Next, it is convenient to define several populations besides $f$, appearing at different stages of an LBM algorithm :

- $f$ : The total population is the most important population because mass, momentum, etc are macroscopic moments of this population.

- $f^{e q}$ : The equilibrium population, here defined as

$$
f_{i}^{e q} \equiv \omega_{i}\left\{\mathcal{H}^{(0)} a^{f^{e q},(0)}+\frac{\mathcal{H}_{i \alpha}^{(1)}}{c_{s}^{2}} a_{\alpha}^{f^{e q},(1)}+\frac{\mathcal{H}_{i \alpha \beta}^{(2)}}{2 c_{s}^{4}} a_{\alpha \beta}^{f^{e q},(2)}+\frac{\mathcal{H}_{i \alpha \beta \gamma}^{(3)}}{6 c_{s}^{6}} a_{\alpha \beta \gamma}^{f^{e q},(3)}+\ldots\right\},
$$

which is usually a Maxwellian projected onto the Hermite basis and properly truncated ${ }^{42}$.

- $f^{n e q}:$ The non-equilibrium population.

$$
f_{i}^{n e q} \equiv f_{i}-f_{i}^{e q}
$$

To ensure $2^{\text {nd }}$ order accuracy ${ }^{30}$, offset distributions $\left(\bar{f}, \bar{f}^{\text {neq }}\right)$ are introduced

- $\bar{f}$ : The modified total population, defined by

$$
\begin{aligned}
\bar{f}_{i} & \equiv f_{i}^{e q}+\left(1+\frac{\Delta t}{2 \tau}\right) f_{i}^{n e q}-\frac{\Delta t}{2} F_{i}, \\
& \equiv f_{i}^{e q}+\bar{f}_{i}^{n e q}-\frac{\Delta t}{2} F_{i},
\end{aligned}
$$


with $F_{i}$ a correction force term defined as

$$
F_{i} \equiv \omega_{i}\left\{\mathcal{H}^{(0)} a^{F,(0)}+\frac{\mathcal{H}_{i \alpha}^{(1)}}{c_{s}^{2}} a_{\alpha}^{F,(1)}+\frac{\mathcal{H}_{i \alpha \beta}^{(2)}}{2 c_{s}^{4}} a_{\alpha \beta}^{F,(2)}+\frac{\mathcal{H}_{i \alpha \beta \gamma}^{(3)}}{6 c_{s}^{6}} a_{\alpha \beta \gamma}^{F,(3)}+\ldots\right\} .
$$

Note that the forcing scheme considered here was presented by ${ }^{56}$.

- $\bar{f}^{\text {neq }}$ : The modified non-equilibrium population ensuring a 2nd order accurate BGK scheme,

$$
\bar{f}_{i}^{n e q} \equiv \bar{f}_{i}-f_{i}^{e q}+\frac{\Delta t}{2} F_{i}
$$

- $f^{c o l}$ : The population at the end of a collide step,

$$
\begin{aligned}
f_{i}^{c o l} & \equiv f_{i}^{e q}+\left(1-\frac{\Delta t}{\bar{\tau}}\right) \bar{f}_{i}^{n e q}+\frac{\Delta t}{2} F_{i}, \\
& \equiv f_{i}^{e q}+\left(1-\frac{\Delta t}{2 \tau}\right) f_{i}^{n e q}+\frac{\Delta t}{2} F_{i},
\end{aligned}
$$

where we used the shorthand $\bar{\tau}=\tau+\Delta t / 2 . f_{i}^{c o l}$ is the population that is streamed during the propagation step. Note that depending on the collision kernel $\bar{f}^{\text {neq }}$ may be replaced by a reconstructed non-equilibrium population $\tilde{f}^{\text {neq }}$.

\section{B. Structure of a generic lattice-Boltzmann scheme}

A LBM model time iteration typically consists of the steps listed in Tab. IV. Let us detail these steps one by one. Although these steps may vary depending on LBM, the general structure remains. At the center of the algorithm lies the collision

$$
f_{i}^{c o l}(t, \boldsymbol{x})=f_{i}^{e q}(t, \boldsymbol{x})+\left(1-\frac{\Delta t}{\bar{\tau}}\right) \bar{f}_{i}^{n e q}(t, \boldsymbol{x})+\frac{\Delta t}{2} F_{i}(t, \boldsymbol{x}),
$$

and streaming steps

$$
\bar{f}_{i}(t+\Delta t, \boldsymbol{x})=f_{i}^{c o l}\left(t, \boldsymbol{x}-\boldsymbol{c}_{\boldsymbol{i}} \Delta t\right) .
$$

They remain generally identical for all LBM as they are the main ingredients responsible for the method's computing efficiency ${ }^{57}$, and low dissipation ${ }^{58}$.

Steps 1 (resp. 7) corresponds to the link between the macroscopic quantities and the population (resp. and back), and vary depending on the model. The force update (step 2) is also model 
Table IV: Generic LBM structure.

\begin{tabular}{lllll}
\hline \hline Step & Description & Input & Output & Equation(s) \\
\hline 1 & Equilibrium update & macroscopic & $f_{i}^{e q}$ & 48 \\
2 & Force update & macroscopic & $F_{i}(t, \boldsymbol{x})$ & 52 \\
3 & Non-equilibrium update & $f_{i}^{e q}, F_{i}(t, \boldsymbol{x})$ & $f_{i}^{\text {neq }}$ & 53 (BGK) \\
4 & Collision & $f_{i}^{e q}, f_{i}^{n e q}, F_{i}(t, \boldsymbol{x})$ & $f_{i}^{c o l}$ & 56 \\
5 & Streaming & $f_{i}^{c o l}\left(t, \boldsymbol{x}-\boldsymbol{c}_{\boldsymbol{i}} \Delta t\right)$ & $\bar{f}_{i}(t+\Delta t, \boldsymbol{x})$ & 57 \\
6 & Coupling with another scheme & $\phi(t, \boldsymbol{x})$ & $\phi(t+\Delta t, \boldsymbol{x})$ & optional \\
7 & Macroscopic quantities update & $\bar{f}_{i}$ & macroscopic & 61 \\
\hline \hline
\end{tabular}

dependent, but is generally computed from the macroscopic variables. Step 3 includes the definition of the collision kernel. Computation of $f_{i}^{\text {neq }}$ will be highly dependent on the collision model, e.g. $\mathrm{BGK}^{22}$, multi-relaxation times models ${ }^{59,60}$, regularized models ${ }^{21}$, and so on.

Step 6 is optional, and corresponds to the resolution of a set of quantities $\phi$, not encompassed in the main LBM population. $\phi$ is a dynamic field, and can correspond to scalars (as in hybrid methods) or additional probability functions (as in double distribution functions). They can represent, e.g.

- Energy ${ }^{31}$

- Species, if the flow is multi-constituent ${ }^{17}$

- an equation for turbulence modeling (e.g. for Spalart-Almaras or $k-\epsilon$ models),

- a liquid mass or volume fraction, for multiphase flows.

While usually presented differently ${ }^{30,39,54,55}$ this structure is shared by all LB schemes using Hermite polynomials. Note that the coupling with the FD/FV/DDF scheme, here identified as step 6, can be performed at any moment in the algorithm except after Step 7, because the macroscopic quantity update may depend on it. 


\section{Taylor expansion}

Now that the basic numerical LB scheme structure has been recast, we can compute its Taylor expansion. We introduce the distribution's Taylor Expansion in space as

$$
f(t, \boldsymbol{x}-\boldsymbol{c} \Delta t)=f(t, \boldsymbol{x})+\sum_{k=1}^{\infty} \frac{(-\Delta t)^{k}}{k !}\left(c_{\alpha_{j}} \frac{\partial}{\partial x_{\alpha_{j}}}\right)^{k} f(t, \boldsymbol{x}),
$$

where $\alpha_{j}$ is a dummy index such that $c_{\alpha_{j}} \partial / \partial x_{\alpha_{j}}=c_{x} \partial / \partial x+c_{y} \partial / \partial y+c_{z} \partial / \partial z$. Taking the $n^{\text {th }}$ order macroscopic moment of the streaming Eq. (57) leads to the moment streaming equation

$$
\Pi_{\alpha_{1} \ldots \alpha_{n}}^{\bar{f}(t+\Delta t, \boldsymbol{x}),(n)}=\Pi_{\alpha_{1} \ldots \alpha_{n}}^{f^{c o l}\left(t, \boldsymbol{x}-c_{\boldsymbol{i}} \Delta t\right),(n)}
$$

Similarly, Eq. (50) can be recast into a moment equation

$$
\Pi_{\alpha_{1} \ldots \alpha_{n}}^{\bar{f}(t+\Delta t, \boldsymbol{x}),(n)}=\Pi_{\alpha_{1} \ldots \alpha_{n}}^{f,(n)}(t+\Delta t, \boldsymbol{x})+\frac{\Delta t}{2}\left(\frac{1}{\tau} \Pi_{\alpha_{1} \ldots \alpha_{n}}^{f^{n e q}(t+\Delta t, \boldsymbol{x}),(n)}-\Pi_{\alpha_{1} \ldots \alpha_{n}}^{F(t+\Delta t, \boldsymbol{x}),(n)}\right) .
$$

Combining Eqs. (59) and (60) finally leads to

$$
\Pi_{\alpha_{1} \ldots \alpha_{n}}^{f,(n)}(t+\Delta t, \boldsymbol{x})=\Pi_{\alpha_{1} \ldots \alpha_{n}}^{f^{c o l}\left(t, \boldsymbol{x}-c_{i} \Delta t\right),(n)}-\frac{\Delta t}{2}\left(\frac{1}{\tau} \Pi_{\alpha_{1} \ldots \alpha_{n}}^{f^{n e q}(t+\Delta t, \boldsymbol{x}),(n)}-\Pi_{\alpha_{1} \ldots \alpha_{n}}^{F(t+\Delta t, \boldsymbol{x}),(n)}\right) .
$$

This equation is the update rule for $(t+\Delta t)$ moments. It is nothing but the LBM numerical scheme written explicitly for the $n^{t h}$ order moment $\Pi_{\alpha_{1} \ldots \alpha_{n}}^{f(t+\Delta t, x),(n)}$. We shall now Taylor expand $\Pi_{\alpha_{1} \ldots \alpha_{n}}^{f^{c o l}\left(t, \boldsymbol{x}-\boldsymbol{c}_{\boldsymbol{i}} \Delta t\right),(n)}$, using Eq. (58),

$$
\Pi_{\alpha_{1} \ldots \alpha_{n}}^{f^{c o l}\left(t, \boldsymbol{x}-c_{i} \Delta t\right),(n)}=\sum_{i}^{q} c_{i \alpha_{1} \ldots} \ldots c_{i \alpha_{n}}\left\{1+\sum_{k=1}^{\infty} \frac{(-\Delta t)^{k}}{k !}\left(c_{i \alpha_{n+j}} \frac{\partial}{\partial x_{\alpha_{n+j}}}\right)^{k}\right\} f_{i}^{c o l}(t, \boldsymbol{x}) .
$$

Using the fact that the discrete velocities $c_{i \alpha_{n}}$ are fixed leads to

$$
\Pi_{\alpha_{1} \ldots \alpha_{n}}^{f^{c o l}\left(t, \boldsymbol{x}-\boldsymbol{c}_{\boldsymbol{i}} \Delta t\right),(n)}=\Pi_{\alpha_{1} \ldots \alpha_{n}}^{f^{c o l}(t, \boldsymbol{x}),(n)}+\sum_{k=1}^{\infty} \frac{(-\Delta t)^{k}}{k !}\left(\frac{\partial}{\partial x_{\alpha_{n+j}}}\right)^{k} \Pi_{\alpha_{1} \ldots \alpha_{n+j}}^{f^{c o l}(t, \boldsymbol{x}),(n+k)} .
$$

It is shown in Appendix A that inserting Eq. (63) into Eq. (61) eventually leads to a secondorder accurate Crank-Nicolson scheme for the continuous equation:

$$
\frac{\partial \Pi_{\alpha_{1} \ldots \alpha_{n}}^{f,(n)}}{\partial t}+\frac{\partial \Pi_{\alpha_{1} \ldots \alpha_{n+1}}^{f,(n+1)}}{\partial x_{\alpha_{n+1}}}=-\frac{1}{\tau} \Pi_{\alpha_{1} \ldots \alpha_{n}}^{f^{n e q},(n)}+\Pi_{\alpha_{1} \ldots \alpha_{n}}^{F,(n)}+O\left(\Delta t^{2}\right)
$$

Note that this equation is only relevant for $q$ independent equations, the isotropy defect (47) leaving higher orders redundant (see Table III). 
Because of the moments cascade, $\Pi_{\alpha_{1} \ldots \alpha_{n}}^{f(t, x),(n+1)}$ is always the flux of $\Pi_{\alpha_{1} \ldots \alpha_{n}}^{f(t, \boldsymbol{x}),(n)}$, allowing moment computation to be algorithmically explicit as long as $-\tau^{-1} \Pi_{\alpha_{1} \ldots \alpha_{n}}^{f^{n e q},(n)}+\Pi_{\alpha_{1} \ldots \alpha_{n}}^{F,(n)}=0$. However, when the collision kernel $-\tau^{-1} \Pi_{\alpha_{1} \ldots \alpha_{n}}^{f^{n e q},(n)}$ or a force term $+\Pi_{\alpha_{1} \ldots \alpha_{n}}^{F,(n)}$ is non-zero one has to solve implicitly Eq. (61) in order to get a second-order accurate scheme.

This layered structure between orders shows that non-equilibrium moments follow their own evolution equation, they are not algebraically enslaved to lower order moments as suggested by the Chapman-Enskog expansion through the scale separation hypothesis. Mandatory conditions for the consistency of LB schemes as NSF solvers will be discussed later for specific kernels.

LBM can be seen as a very smart change of variable from macroscopic moments to distribution functions. First the macroscopic information is stored inside the Hermite basis through $f^{c o l}$ (first change of variable from $\Pi^{(n)}$ to $f$ ). Then the transport is performed in the distribution space during the streaming, followed by the macroscopic reconstruction that filters only the relevant information for each macroscopic moment $\Pi^{(n)}$ (second change or variable from $f$ to $\left.\Pi^{(n)}\right)$, prompting us to draw a parallel between classical CFD and LBM, which is now only seen as a numerical macroscopic solver.

This shows that even if the CE expansion leads to the desired set of macroscopic equation it does not leads to the necessary result that would allow us to fully understand what solves LBMs, namely Eq. (64), a Grad- $q$ system of equations.

In the following we will illustrate the Taylor expansion on three different LBMs, namely the athermal, density-based recursive regularized (RR- $\rho$ ) and pressure-based recursive regularized (RR-p) models. We shall also demonstrate that although Eq. (64) could have been easily guessed from Eq. (42), spurious terms will appear during the coupling between LB algorithm and other numerical schemes. Because these terms are purely stemming from the numerical coupling they are out of the scope of the CE expansion.

\section{ATHERMAL LBM}

The last section showed that each macroscopic moment follows its own evolution equation (64), advocating a change of paradigm. Instead of considering LBM as a kinetic solver let us consider it as a Grad- $q$ solver for an extended set of thermo-hydrodynamic equations. Some of them are desired conservation laws such as mass and momentum conservation, others corresponds to higher order equations in the finite hierarchy of $q$ equations related to the lattice 
$D d Q q$. Therefore, $f$ and all other populations previously defined lose their kinetic meaning and are now merely seen as temporary variables in the macroscopic CFD solver known as "LBM".

\section{A. Athermal numerical scheme}

For the sake of clarity we first apply the proposed Taylor expansion on the classical athermal LBM on standard lattices ${ }^{30}$ with a force term specifically designed to get rid of the well known $O\left(\mathrm{Ma}^{3}\right)$ error detailed in Table III. This model, traditionally said to be athermal in the LB literature, is often used in practice to solve isothermal flows. Following the new paradigm we define the initial solution simply by initial macroscopic fields $\rho(t, \boldsymbol{x}), u_{\alpha}(t, \boldsymbol{x})$.From this initial condition we would like to find a LB algorithm that predicts $\rho(t+\Delta t, \boldsymbol{x})$ and $u_{\alpha}(t+\Delta t, \boldsymbol{x})$ following an approximate Navier-Stokes system, hopefully matching Eqs. $(1,2)$. Let us now detail step-by-step the algorithm proposed in Table IV, applied to the classical athermal LBM.

- Step 1 : Equilibrium construction Because we restrict ourselves to standard lattices, some third-order Hermite polynomials $\mathcal{H}_{i}^{(3)}$ do not belong to the Hermite basis. For this reason we do not expand further than the third order, isotropy defects being corrected by an appropriate force term. The equilibrium reads

$$
f_{i}^{e q}=\omega_{i}\left\{\mathcal{H}^{(0)} \rho+\frac{\mathcal{H}_{i \alpha}^{(1)}}{c_{s}^{2}} \rho u_{\alpha}+\frac{\mathcal{H}_{i \alpha \beta}^{(2)}}{2 c_{s}^{4}}\left[\rho u_{\alpha} u_{\beta}\right]+\frac{\mathcal{H}_{i \alpha \beta \gamma}^{(3)}}{6 c_{s}^{6}}\left[\rho u_{\alpha} u_{\beta} u_{\gamma}\right]\right\} .
$$

- Step 2 : Force construction The forcing population is extended to second order,

$$
F_{i} \equiv \omega_{i}\left\{\mathcal{H}^{(0)} a^{F,(0)}+\frac{\mathcal{H}_{i \alpha}^{(1)}}{c_{s}^{2}} a_{\alpha}^{F,(1)}+\frac{\mathcal{H}_{i \alpha \beta}^{(2)}}{2 c_{s}^{4}} a_{\alpha \beta}^{F,(2)}\right\},
$$

with its Hermite moments defined as

$$
\begin{aligned}
a_{\alpha \beta}^{F,(2)} & =-\frac{\partial D_{\alpha \beta \gamma}^{f e q},(3)}{\partial x_{\gamma}}+\rho c_{s}^{2} \frac{2}{3} \frac{\partial u_{\gamma}}{\partial x_{\gamma}} \delta_{\alpha \beta}+\rho \mathcal{F}_{\alpha} u_{\beta}+\rho \mathcal{F}_{\beta} u_{\alpha}-\dot{m} u_{\alpha} u_{\beta}, \\
a_{\alpha}^{F,(1)} & =\rho \mathcal{F}_{\alpha}, \\
a^{F,(0)} & =\dot{m},
\end{aligned}
$$

with $D_{\alpha \beta \gamma}^{f^{e q},(3)}$ the isotropy defect of the equilibrium population, related to the lattice and the particular equilibrium function. Remember that $\dot{m}$ and $\mathcal{F}_{\alpha}$ correspond respectively to a mass source and volume force in the macroscopic equations $(1,2)$. 
- Step 3 : Non-equilibrium construction Using a BGK kernel, the non-equilibrium population is obtained as

$$
\bar{f}_{i}^{\text {neq }}=\bar{f}_{i}-f_{i}^{e q}+\frac{\Delta t}{2} F_{i}
$$

Otherwise, following a particular choice of collision kernel construct $\tilde{f}_{i}^{n e q}$ and set

$$
\bar{f}_{i}^{\text {neq }}=\tilde{f}_{i}^{\text {neq }} .
$$

- Step 4 : Collision With $f_{i}^{e q}, F_{i}$ and $\bar{f}_{i}^{n e q}$ built in the previous steps, compute the collided population $f_{i}^{c o l}$ as

$$
f_{i}^{c o l}(t, \boldsymbol{x})=f_{i}^{e q}(t, \boldsymbol{x})+\left(1-\frac{\Delta t}{\bar{\tau}}\right) \bar{f}_{i}^{n e q}(t, \boldsymbol{x})+\frac{\Delta t}{2} F_{i}(t, \boldsymbol{x})
$$

- Step 5 : Streaming Transport the populations according to

$$
\bar{f}_{i}(t+\Delta t, \boldsymbol{x})=f_{i}^{c o l}\left(t, \boldsymbol{x}-\boldsymbol{c}_{\boldsymbol{i}} \Delta t\right) .
$$

- Step 6 : Coupling update No coupling is necessary in the athermal case, but this stage can be used to transport passive scalars ${ }^{61-63}$.

- Step 7 : Update macroscopic variables Using the macroscopic update rule Eq. (61) for $n=0,1,2$ respectively leads to

$$
\begin{aligned}
\rho(t+\Delta t, \boldsymbol{x}) & =\sum_{i}^{q} \bar{f}_{i}(t+\Delta t, \boldsymbol{x})+\frac{\Delta t}{2} \dot{m}(t+\Delta t, \boldsymbol{x}) \\
\rho u_{\alpha}(t+\Delta t, \boldsymbol{x}) & =\sum_{i}^{q} c_{i \alpha} \bar{f}_{i}(t+\Delta t, \boldsymbol{x})+\frac{\Delta t}{2}\left[\rho \mathcal{F}_{\alpha}\right](t+\Delta t, \boldsymbol{x}) \\
\Pi_{\alpha \beta}^{f,(2)}(t+\Delta t, \boldsymbol{x}) & =\Pi_{\alpha \beta}^{\bar{f}^{(t+\Delta t, \boldsymbol{x}),(2)}-\frac{\Delta t}{2}}\left(\frac{1}{\tau} \Pi_{\alpha \beta}^{f^{n e q}(t+\Delta t, \boldsymbol{x}),(2)}-\Pi_{\alpha \beta}^{F(t+\Delta t, \boldsymbol{x}),(2)}\right) .
\end{aligned}
$$

By splitting $\Pi_{\alpha \beta}^{f,(2)}(t+\Delta t, \boldsymbol{x})$ into its equilibrium and non-equilibrium parts, the above leads to the stress-tensor scheme

$$
\left(1+\frac{\Delta t}{2 \tau}\right) \Pi_{\alpha \beta}^{f^{n e q}(t+\Delta t, \boldsymbol{x}),(2)}=\Pi_{\alpha \beta}^{\bar{f}(t+\Delta t, \boldsymbol{x}),(2)}-\Pi_{\alpha \beta}^{f^{e q}(t+\Delta t, \boldsymbol{x}),(2)}+\frac{\Delta t}{2} \Pi_{\alpha \beta}^{F(t+\Delta t, \boldsymbol{x}),(2)}
$$




\section{B. Continuous equivalent equations}

Now that macroscopic quantities, namely mass, velocity and stress-tensor $\rho(t+\Delta t, \boldsymbol{x}), u_{\alpha}(t+$ $\Delta t, \boldsymbol{x})$ and $\Pi_{\alpha \beta}^{f^{n e q},(2)}(t+\Delta t, \boldsymbol{x})$ have been explicitly updated let us analyze the equivalent continuous equations of System $(74,75,77)$ and compare it with the target set of equations Eqs. $(1,2)$ with $p=\rho c_{s}^{2}(5)$.

Using the continuous limit Eq. (64) of the LB scheme leads to an extended Grad- $q$ system that conditionally approximates the athermal Navier-Stokes:

$$
\begin{gathered}
\frac{\partial \rho}{\partial t}+\frac{\partial \rho u_{\beta}}{\partial x_{\beta}}=\dot{m} \\
\frac{\partial \rho u_{\alpha}}{\partial t}+\frac{\partial\left[\rho u_{\alpha} u_{\beta}+p \delta_{\alpha \beta}+\Pi_{\alpha \beta}^{f^{n e q},(2)}\right]}{\partial x_{\beta}}=\rho \mathcal{F}_{\alpha}, \\
\frac{\partial \Pi_{\alpha \beta}^{f,(2)}}{\partial t}+\frac{\partial\left[\Pi_{\alpha \beta \gamma}^{f,(3)}-D_{\alpha \beta \gamma}^{f,(3)}\right]}{\partial x_{\gamma}}=-\frac{1}{\tau} \Pi_{\alpha \beta}^{f^{n e q},(2)}+\Pi_{\alpha \beta}^{F,(2)} .
\end{gathered}
$$

where the lattice-dependent isotropy defect $D_{\alpha \beta \gamma}^{f,(3)}$ can be found in Table III.

From this system we can infer that $-\Pi_{\alpha \beta}^{f^{n e q},(2)}$ is the effective stress tensor. We also see that contrary to the usual Navier-Stokes equations this system has an evolution equation for the stresstensor, Eq. (80). This evolution equation involves $\Pi_{\alpha \beta \gamma}^{f^{n e q},(3)}$ through $\Pi_{\alpha \beta \gamma}^{f,(3)}$, which depends on higher order contributions and is assumed negligible by the CE expansion. The term $\partial \Pi_{\alpha \beta}^{f^{e q},(2)} / \partial t$ hidden inside Eq. (80) can be replaced using Eq. (4). Using the second order moment of the equilibrium population $(67,69)$ in Eq. $(80)$ and the athermal equation of state (5) finally leads to

$$
\begin{gathered}
-\Pi_{\alpha \beta}^{f^{n e q},(2)}=\tau \rho c_{s}^{2}\left[\frac{\partial u_{\alpha}}{\partial x_{\beta}}+\frac{\partial u_{\beta}}{\partial x_{\alpha}}-\delta_{\alpha \beta} \frac{2}{3} \frac{\partial u_{\gamma}}{\partial x_{\gamma}}\right] \\
+\tau\left[\frac{\partial \Pi_{\alpha \beta}^{f^{n e q},(2)}}{\partial t}+\frac{\partial\left[\Pi_{\alpha \beta \gamma}^{f^{n e q},(3)}-D_{\alpha \beta \gamma}^{f^{n e q},(3)}\right]}{\partial x_{\gamma}}\right]-\tau\left[u_{\alpha} \frac{\partial \Pi_{\beta \gamma}^{f^{n e q},(2)}}{\partial x_{\gamma}}+u_{\beta} \frac{\partial \Pi_{\alpha \gamma}^{f^{n e q},(2)}}{\partial x_{\gamma}}\right]
\end{gathered}
$$

with $\tau \rho c_{s}^{2}=\mu$ obtained by identification with the usual definition of the stress tensor (3). Note that the effect of the collision kernel is entirely hidden inside $\Pi_{\alpha \beta \gamma}^{f^{n e q},(3)}$. When using a simple BGK collision step $\Pi_{\alpha \beta \gamma}^{f^{n e q},(3)}$ is enslaved to higher order unphysical contributions because of the lack of isotropy that leads to an under-resolved finite hierarchy of equations in the velocity space.

This last equation is not algebraic as the truncated CE expansion asserts but rather an evolution equation for the unknown $\Pi_{\alpha \beta}^{f^{n e q},(2)}$. 


\section{Domain of validity in term of dimensionless numbers}

The next step is to demonstrate in which cases the Lattice Boltzmann stress-tensor equation is consistent with a Navier-Stokes stress tensor (80)

$$
-\Pi_{\alpha \beta}^{f^{n e q},(2)} \approx \mathcal{T}_{\alpha \beta}=\mu\left[\frac{\partial u_{\alpha}}{\partial x_{\beta}}+\frac{\partial u_{\beta}}{\partial x_{\alpha}}-\frac{2 \delta_{\alpha \beta}}{3} \frac{\partial u_{\gamma}}{\partial x_{\gamma}}\right] .
$$

To that end, let us nondimensionalize Eq. (81). First we need to identify what is the shortest physical time scale $t_{s}$, corresponding to the fastest and dominant physical phenomenon. Depending on the situation, mainly two relevant candidates exist: the viscous timescale $t_{\mu}=\rho L_{0}^{2} / \mu$ and the convective timescale $t_{c}=L_{0} / U_{0}$.

If the shortest timescale is $t_{s}$, then the appropriate nondimensionalization reads

$$
\begin{array}{r}
\frac{\partial}{\partial t}=\frac{1}{t_{s}} \frac{\partial}{\partial t^{*}}, \quad \frac{\partial}{\partial x}=\frac{1}{L_{0}} \frac{\partial}{\partial x^{*}}, \\
\Pi_{\alpha \beta}^{f^{n e q},(2)}=\Pi_{0} \Pi_{\alpha \beta}^{*, f^{n e q},(2)}, \quad \Pi_{\alpha \beta \gamma}^{f^{n e q},(3)}=Q_{0} \Pi_{\alpha \beta \gamma}^{*, f^{n e q},(3)}, \\
u=U_{0} u^{*}, \quad \rho=\rho_{0} \rho^{*}, \quad T=T_{0} T^{*} .
\end{array}
$$

where * superscript quantities are $O(1)$ and non-dimensional. Applying this change of variable,

$$
\begin{gathered}
-\Pi_{\alpha \beta}^{*, f^{n e q},(2)}=\mathcal{T}_{\alpha \beta}^{*}+\frac{\mu Q_{0}}{\rho_{0} c_{s}^{2} L_{0} \Pi_{0}} \frac{1}{\rho^{*}} \frac{\partial\left[\Pi_{\alpha \beta \gamma}^{*, f^{n e q},(3)}-D_{\alpha \beta \gamma}^{*, f^{n e q},(3)}\right]}{\partial x_{\gamma}^{*}} \\
+\frac{\widetilde{M}^{2}}{\operatorname{Re}} \frac{1}{\rho^{*}}\left[u_{\alpha}^{*} \frac{\partial \Pi_{\beta \gamma}^{*, f^{n e q},(2)}}{\partial x_{\gamma}^{*}}+u_{\beta}^{*} \frac{\partial \Pi_{\alpha \gamma}^{*, f^{n e q},(2)}}{\partial x_{\gamma}^{*}}\right]+\frac{\mu}{\rho_{0} c_{s}^{2} t_{s}} \frac{1}{\rho^{*}} \frac{\partial \Pi_{\alpha \beta}^{*, f^{n e q},(2)}}{\partial t^{*}},
\end{gathered}
$$

where the Reynolds number $R e=t_{\mu} / t_{c}$ and athermal Mach number

$$
\widetilde{M a}=U_{0} / c_{s}
$$

have been used. This implicitly means that in the athermal case $\widetilde{M} a$ is enslaved to the CFL number ${ }^{24}$ because $c_{s}=\Delta x /(\sqrt{3} \Delta t)$, leading to

$$
C F L=\frac{U_{0}+c_{s}}{\Delta x / \Delta t}=\frac{\widetilde{M} a+1}{\sqrt{3}} .
$$

Note that the stability criterion CFL $\leq 1$ boils down to the usual athermal Mach limit $\widetilde{M a} \leq$ $\sqrt{3}-1 \approx 0.732$, which is consistent with previous studies ${ }^{64,65}$. If the convective scaling is chosen the stress-tensor becomes

$$
-\Pi_{\alpha \beta}^{*, f^{n e q},(2)}=\mathcal{T}_{\alpha \beta}^{*}+O\left(\frac{\mu Q_{0}}{\rho_{0} c_{s}^{2} L_{0} \Pi_{0}}\right)+O\left(\frac{\widetilde{M a}^{2}}{R e}\right) .
$$


The condition of consistency on LBMs is that prefactors should be negligible quantities to verify our initial assumption Eq. (82). The last term of the right hand side can be neglected for the diffusive and convective timescale respectively if $\widetilde{M a}^{2} / R e^{2}$ and $\widetilde{M}^{2} / R e$ are small enough. On the other hand the factor $\mu Q_{0} / \rho_{0} c_{s}^{2} L_{0} \Pi_{0}$ corresponding to the scaling between the third order non-equilibrium and second order non-equilibrium is not generally trivial. Because the isotropy

defect completely modifies the convective term in the evolution equation of $\Pi_{\alpha \beta \gamma}^{f^{n e q}}$,(3) , it leads to an evolution equation whose physical meaning is unclear. More importantly, this unphysical evolution equation can be a source of instabilities ${ }^{66}$. However, when the recursive regularized kernel $^{49}$ is used, $Q_{0}$ is known and the stress-tensor becomes

$$
-\Pi_{\alpha \beta}^{*, f^{n e q},(2)}=\mathcal{T}_{\alpha \beta}^{*}+O\left(\frac{\widetilde{M a}^{2}}{R e}\right)
$$

One sees here that the usual "small Knudsen" assumption is not even sufficient because Kn.Ma terms appeared in the scaling analysis. To get back the proper NSF stress-tensor one have to carefully analyze one by one each of these spurious terms. Additionally the scaling $Q_{0}$ is related to the heat flux, suggesting that the Prandtl number Pr should also intervene in the range of consistency of LBMs.

The Taylor expansion showed us that the consistency condition was not as simple as the CE expansion suggests. The small Knudsen assumption is not enough and both the choice of the lattice and the collision kernel changes the consistency defect in Eq. (90). More discrete velocities mean that the isotropy defect is pushed away from the Navier-Stokes and the stress-tensor equation, but it also means that more unphysical equations are also solved. Those equations are likely to modify or even undermine the validity of the solution, such as for Burnett-like systems.

\section{COLLISION KERNEL}

Now that we analyzed a simple athermal BGK LB scheme let us discuss the collision kernel by reviewing a sample of techniques that can be used to increase robustness of LBMs. From the Taylor expansion we've seen that mass and momentum conservation equations were correctly discretized up to $O\left(\Delta t^{2}\right)$ errors by the LB scheme. On the other hand, the system is not closed through an algebraic constitutive equation as in usual CFD solvers. Instead we inherit Eq (81) 
from the hierarchy of moments. Rearranging its terms leads to

$$
\frac{\partial \Pi_{\alpha \beta}^{f^{n e q},(2)}}{\partial t}+\frac{\partial\left[\Pi_{\alpha \beta \gamma}^{f^{n e q},(3)}-D_{\alpha \beta \gamma}^{f^{n e q},(3)}\right]}{\partial x_{\gamma}}-u_{\alpha} \frac{\partial \Pi_{\beta \gamma}^{f^{n e q},(2)}}{\partial x_{\gamma}}-u_{\beta} \frac{\partial \Pi_{\alpha \gamma}^{f^{n e q},(2)}}{\partial x_{\gamma}}=-\frac{1}{\tau}\left(\Pi_{\alpha \beta}^{f^{n e q},(2)}+\mathcal{T}_{\alpha \beta}\right),
$$

where $\mathcal{T}_{\alpha \beta}(3)$ is the physical stress tensor. Higher order contributions $\Pi_{\alpha \beta \gamma}^{f^{n e q},(3)}$, as already discussed, do not necessarily match a physical behavior, especially for standard lattices because of isotropy defects. Therefore, a question that could drive us towards the use of a particular kernel is a correct modeling of the stress tensor by Eq. (91). Being the only moment that does not appear in the hydrodynamic equations Eqs. (78-79) the non-equilibrium tensor $\Pi_{\alpha \beta \gamma}^{f^{n e q} \text {,(3) }}$ is our only degree of freedom to modify the modeling of Eq. (91) towards a physically meaningful stress tensor transport equation. For example with a BGK collision operator, $\Pi_{\alpha \beta \gamma}^{f^{n e q} \text {,(3) }}$ purely stems from higher order non-hydrodynamic equations. In this case, assuming that the lattice is large enough such that $D_{\alpha \beta \gamma}^{f^{n e q},(3)}=0$ (this property is only enforced on some non-diagonal components of $\Pi_{\alpha \beta \gamma}^{(3)}$ for standard lattices) we can write for example the $\Pi_{x x}^{f^{n e q},(2)}$ evolution equation,

$$
\frac{\partial \Pi_{x x}^{f^{n e q},(2)}}{\partial t}-2 u_{x} \frac{\partial \Pi_{x y}^{f^{n e q},(2)}}{\partial x_{\gamma}}=-\frac{1}{\tau}\left(\Pi_{x x}^{f^{n e q},(2)}+\mathcal{T}_{x x}\right)-\frac{\partial \Pi_{x x y}^{f^{n e q},(3)}}{\partial x_{y}} .
$$

In the left hand side we recognize a transport term in the $x$ direction with an unexpected backward propagation $-2 u_{x}$ while the first term in the right hand side is a relaxation term that steers the variable $\Pi_{x x}^{f^{n e q},(2)}$ towards the expected $\mathcal{T}_{x x}$. The second term in the right hand side is the coupling with higher-order non-hydrodynamic moments.

\section{A. Effect of regularized kernels}

A particularly efficient way to control the time evolution of the stress tensor was identified in the regularized and recursive regularized collision kernels. The simple regularization ${ }^{67,68}$ simply discards $\Pi_{\alpha \beta \gamma}^{\bar{f}^{n e q}}$,(3) during the collision leading to a filtered non-equilibrium population

$$
\begin{gathered}
\bar{f}_{i}^{\mathrm{neq}}=\omega_{i} \frac{\mathcal{H}_{i \alpha \beta}^{(2)}}{2 c_{s}^{4}} \Pi_{\alpha \beta}^{\bar{f}^{\mathrm{neq}},(2)}, \\
\Pi_{\alpha \beta}^{\bar{f}^{\mathrm{neq}},(2)}=\sum_{i}^{q} c_{i \alpha} c_{i \beta}\left(\bar{f}_{i}-f_{i}^{\mathrm{eq}}+\frac{\Delta t}{2} F_{i}\right)
\end{gathered}
$$

allowing those filtered moments to be compartmentalized from the hydrodynamic moments, and effectively canceling the last term of Eq. (92). 


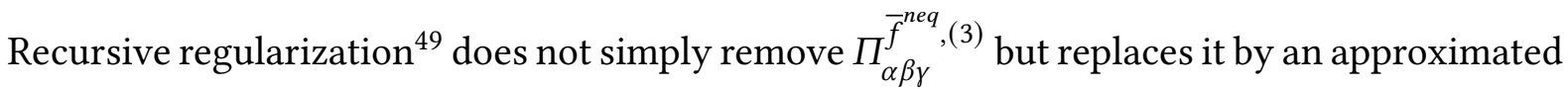
value obtained from the CE expansion,

$$
\bar{f}_{i}^{\text {neq }}=\omega_{i}\left\{\frac{\mathcal{H}_{i \alpha \beta}^{(2)}}{2 c_{s}^{4}} \Pi_{\alpha \beta}^{\bar{f}^{\mathrm{neq}},(2)}+\frac{\mathcal{H}_{i \alpha \beta \gamma}^{(3)}}{6 c_{s}^{6}}\left(u_{\alpha} \Pi_{\beta \gamma}^{\bar{f}^{\mathrm{neq}},(2)}+u_{\beta} \Pi_{\gamma \alpha}^{\bar{f}^{\mathrm{neq}},(2)}+u_{\gamma} \Pi_{\alpha \beta}^{\bar{f}^{\mathrm{neq}},(2)}\right)\right\},
$$

leading to a new evolution equation,

$$
\frac{\partial \Pi_{x x}^{f^{n e q},(2)}}{\partial t}+\frac{\partial u_{\gamma} \Pi_{x x}^{f^{n e q},(2)}}{\partial x_{y}}+2 \Pi_{x y}^{f^{n e q},(2)} \frac{\partial u_{x}}{\partial x_{y}}=-\frac{1}{\tau}\left(\Pi_{x x}^{f^{n e q},(2)}+\mathcal{T}_{x x}\right)
$$

that now exhibits a forward transport, which may explain why high velocity flows are more stable $^{49}$ with this regularized collision kernel.

\section{B. Effect of the Hybrid Recursive Regularization}

An extension of the recursive regularization was developed ${ }^{21}$ by introducing $\sigma \in[0,1]$ into the non-equilibrium reconstruction Eq. (95) as

$$
\Pi_{\alpha \beta}^{\bar{f}^{\mathrm{neq}},(2)}=\sigma \sum_{i=1}^{q} c_{i \alpha} c_{i \beta}\left(\bar{f}_{i}-f_{i}^{e q}+\frac{\Delta t}{2} F_{i}\right)-(1-\sigma) \rho c_{s}^{2} \bar{\tau}\left[\frac{\partial u_{\alpha}}{\partial x_{\beta}}+\frac{\partial u_{\beta}}{\partial x_{\alpha}}-\frac{2 \delta_{\alpha \beta}}{3} \frac{\partial u_{\gamma}}{\partial x_{\gamma}}\right]_{F D}
$$

where the last term is evaluated from a finite difference scheme. It has been shown ${ }^{21}$ that this modification leads, for $\sigma<1$, to the introduction of a numerical hyperviscosity in the momentum equation.

In light of the previous Section, an alternative explanation for the enhanced stability is that $\Pi_{\alpha \beta}^{f^{\text {neq, }}(2)}$ may deviate from its target value $\mathcal{T}_{\alpha \beta}$. Using $0<\sigma<1$ (resp. $\sigma=0$ ) as a weighting

parameter is equivalent to a partial (resp. total) reset of $\Pi_{\alpha \beta}^{f^{\text {neq, (2) }}}$ to its fully relaxed value $\mathcal{T}_{\alpha \beta}$ at the end of each time step, leading to a stronger steering of $\Pi_{\alpha \beta}^{f^{\text {neq }},(2)}$ towards $\mathcal{T}_{\alpha \beta}$ by the resulting LB scheme.

\section{Trace of the stress tensor}

The pressure work is of paramount importance in compressible flows and was already emphasized as a major source of instabilities for thermal $\mathrm{LBMs}^{64}$. Because the non-equilibrium moment $\Pi_{\alpha \beta}^{f^{\text {neq }},(2)}$ is the effective stress tensor in LBMs, any spurious term appearing on its trace will act 
as a spurious pressure in momentum equation. Therefore, artificially enforcing a traceless stress tensor ${ }^{7}$,

$$
\Pi_{\alpha \beta}^{\bar{f}^{\mathrm{neq}},(2)}=\sum_{i}^{q}\left[c_{i \alpha} c_{i \beta}-\frac{\delta_{\alpha \beta}}{d} c_{i \gamma} c_{i \gamma}\right]\left(\bar{f}_{i}-f_{i}^{\mathrm{eq}}+\frac{\Delta t}{2} F_{i}\right)
$$

during the collision allows to get rid of this spurious pressure.

Because $\rho$ and $\rho u_{\alpha}$ are conserved moments in LBMs, the collision kernel has no direct impact on their numerical schemes. But we've seen in this section that a choice of collision kernel impacts the closure for the $\Pi_{\alpha \beta}^{f^{\text {neq }},(2)}$ equation. Therefore, a choice of collision kernel is a choice of closure for non-equilibrium moments. For this reason, we believe the present method to be a good candidate to analyze the LBM closure, and design future collision kernels.

\section{THERMAL RR-p}

Now that we analyzed both the athermal model and the collision kernel, let us analyze two thermal models using the recursive regularized collision kernel. These models are of particular interest to highlight advantages of the Taylor expansion over CE as it has both a coupling with another algorithm and a complex collision kernel.

Let us start with the recursive regularized pressure-based model (RR-p), presented earlier this year $^{7}$, to simulate both compressible flows and reactive flows ${ }^{18}$.

The main feature in this model is that the equilibrium population is chosen so that the 0th order moment of the equilibrium population is a normalized pressure, the rest of the equilibrium is similar to the athermal equilibrium distribution Eq. (65). It leads to an unphysical mass conservation equation that is mended by a correcting term that explicitly uses the Taylor expansion, which is by itself a sufficient reason to analyze this model through the scope of the Taylor expansion instead of the CE expansion.

Since standard lattices are not able to recover accurately the second-order moment corresponding to energy, the energy conservation (6) is solved separately, as $\phi$ in Step 6 of Table IV. Also note that the athermal equation of state is replaced, from here on, by the perfect gas equation of state (7). 


\section{A. RR-p numerical scheme}

let us assume that we have a consistent and convergent explicit scheme for Eq. (6), expressed as

$$
[\rho E](t+\Delta t, \boldsymbol{x})=[\rho E](t, \boldsymbol{x})+\Delta t \square_{\rho E}(t, \boldsymbol{x})+\mathcal{O}\left(\Delta t^{m}\right)
$$

with $m \geq 1$ and $\square_{\rho E}$ an operator that represents everything but the time derivative in Eq. (6). We shall now detail the step-by-step algorithm leading to the RR-p model.

- Step 1 : Equilibrium construction The equilibrium is expanded as

$$
f_{i}^{e q}=\omega_{i}\left\{\mathcal{H}^{(0)} \rho \theta+\frac{\mathcal{H}_{i \alpha}^{(1)}}{c_{s}^{2}} \rho u_{\alpha}+\frac{\mathcal{H}_{i \alpha \beta}^{(2)}}{2 c_{s}^{4}}\left[\rho u_{\alpha} u_{\beta}\right]+\frac{\mathcal{H}_{i \alpha \beta \gamma}^{(3)}}{6 c_{s}^{6}}\left[\rho u_{\alpha} u_{\beta} u_{\gamma}\right]\right\} .
$$

Note the slight modification in the 0th order, with this model the density $\rho$ is replaced by a normalized pressure

$$
\rho \theta=p / c_{s}^{2}
$$

- Step 2 : Force construction The forcing population is simply extended to second order,

$$
F_{i} \equiv \omega_{i}\left\{\mathcal{H}^{(0)} a^{F,(0)}+\frac{\mathcal{H}_{i \alpha}^{(1)}}{c_{s}^{2}} a_{\alpha}^{F,(1)}+\frac{\mathcal{H}_{i \alpha \beta}^{(2)}}{2 c_{s}^{4}} a_{\alpha \beta}^{F,(2)}\right\}
$$

with its Hermite moments defined as

$$
\begin{aligned}
a_{\alpha \beta}^{F,(2)} & =c_{s}^{2} u_{\alpha} \frac{\partial \rho(1-\theta)}{\partial x_{\beta}}+c_{s}^{2} u_{\beta} \frac{\partial \rho(1-\theta)}{\partial x_{\alpha}} \\
& +\delta_{\alpha \beta} \rho c_{s}^{2} \frac{2}{3} \frac{\partial u_{\gamma}}{\partial x_{\gamma}}-c_{s}^{2} \delta_{\alpha \beta} \frac{\partial \rho(1-\theta)}{\partial t}-\frac{\partial D_{\alpha \beta \gamma}^{f^{e q},(3)}}{\partial x_{\gamma}} \\
& +\rho \mathcal{F}_{\alpha} u_{\beta}+\rho \mathcal{F}_{\beta} u_{\alpha}-\dot{m} u_{\alpha} u_{\beta}, \\
a_{\alpha}^{F,(1)} & =\rho \mathcal{F}_{\alpha}, \\
a^{F,(0)} & =\dot{m} .
\end{aligned}
$$

- Step 3 : Non-equilibrium construction Though this particular pressure-based coupling does not presuppose the use of any particular collision kernel we chose the recursive regularized collision operator ${ }^{7}$. The non-equilibrium population $\bar{f}_{i}^{\text {neq }}$ is then reconstructed following Eq. (95). 
- Step 4 : Collision Thanks to previous steps $f_{i}^{e q}, F_{i}$ and $\bar{f}_{i}^{\text {neq }}$ have been built, compute the collided population $f_{i}^{c o l}$ such that

$$
f_{i}^{c o l}(t, \boldsymbol{x})=f_{i}^{e q}(t, \boldsymbol{x})+\left(1-\frac{\Delta t}{\bar{\tau}}\right) \bar{f}_{i}^{n e q}(t, \boldsymbol{x})+\frac{\Delta t}{2} F_{i}(t, \boldsymbol{x}) .
$$

- Step 5 : Streaming Shift the populations according to

$$
\bar{f}_{i}(t+\Delta t, \boldsymbol{x})=f_{i}^{c o l}\left(t, \boldsymbol{x}-\boldsymbol{c}_{\boldsymbol{i}} \Delta t\right)
$$

- Step 6 : Coupling update Here simply solve the explicit numerical scheme Eq. (99) to get $[\rho E](t+\Delta t, \boldsymbol{x})$.

- Step 7 : Update macroscopic When applied to $n=0$ the macroscopic update rule Eq. (61) usually gives us the updated density $\rho(t+\Delta t, \boldsymbol{x})$. But here because we modified the equilibrium distribution it leads to an updated pressure $\left(p^{*} / c_{s}^{2}\right)(t+\Delta t, \boldsymbol{x})$ such that

$$
\left(\frac{p^{*}}{c_{s}^{2}}\right)(t+\Delta t, \boldsymbol{x})=\Pi^{\bar{f}(t+\Delta t, \boldsymbol{x}),(0)}+\frac{\Delta t}{2} \dot{m}(t+\Delta t, \boldsymbol{x}) .
$$

which is equivalent to an unphysical equation for the pressure, $\partial\left(p^{*} / c_{s}^{2}\right) / \partial t+\partial \rho u_{\beta} / \partial x_{\beta}=\dot{m}$, see Eq. (64) for $n=0$. To recover the correct mass conservation equation it is mandatory to modify the 0 th order update rule such as :

$$
\rho(t+\Delta t, \boldsymbol{x})=\Pi^{\bar{f}(t+\Delta t, \boldsymbol{x}),(0)}+\rho(t, \boldsymbol{x})[1-\theta]+\frac{\Delta t}{2} \dot{m}(t+\Delta t, \boldsymbol{x})
$$

The update rule for $n=1,2$ remains unchanged,

$$
\begin{aligned}
\rho u_{\alpha}(t+\Delta t, \boldsymbol{x}) & =\sum_{i}^{q} c_{i \alpha} \bar{f}_{i}(t+\Delta t, \boldsymbol{x})+\frac{\Delta t}{2}\left[\rho \mathcal{F}_{\alpha}\right](t+\Delta t, \boldsymbol{x}), \\
\left(1+\frac{\Delta t}{2 \tau}\right) \Pi_{\alpha \beta}^{f^{n e q}(t+\Delta t, \boldsymbol{x}),(2)} & =\Pi_{\alpha \beta}^{\bar{f}(t+\Delta t, \boldsymbol{x}),(2)}-\Pi_{\alpha \beta}^{f^{e q}(t+\Delta t, \boldsymbol{x}),(2)}+\frac{\Delta t}{2} \Pi_{\alpha \beta}^{F(t+\Delta t, \boldsymbol{x}),(2)} .
\end{aligned}
$$

Then compute $T(t+\Delta t, \boldsymbol{x})$, from $[\rho E](t+\Delta t, \boldsymbol{x}), u_{\alpha}(t+\Delta t, \boldsymbol{x})$ and $\rho(t+\Delta t, \boldsymbol{x})$.

\section{B. Continuous equivalent equations}

Now that macroscopic quantities, namely mass, velocity, stress-tensor and temperature, $\rho(t+$ $\Delta t, \boldsymbol{x}), u_{\alpha}(t+\Delta t, \boldsymbol{x}), \Pi_{\alpha \beta}^{f^{n e q},(2)}(t+\Delta t, \boldsymbol{x})$ and $T(t+\Delta t, \boldsymbol{x})$ have been explicitly updated let us analyze 
the equivalent continuous equations of the system Eqs. $(109,110,111,99)$ and compare it with the target set of equations Eqs. $(1,2,6)$. The Taylor expansion leads to the system of equations

$$
\begin{gathered}
\frac{\partial \rho}{\partial t}+\frac{\partial \rho u_{\beta}}{\partial x_{\beta}}=\dot{m}, \\
\frac{\partial \rho u_{\alpha}}{\partial t}+\frac{\partial\left[\rho u_{\alpha} u_{\beta}+p \delta_{\alpha \beta}+\Pi_{\alpha \beta}^{f^{n e q},(2)}\right]}{\partial x_{\beta}}=\rho \mathcal{F}_{\alpha}, \\
\frac{\partial \Pi_{\alpha \beta}^{f,(2)}+\frac{\partial\left[\Pi_{\alpha \beta \gamma}^{f,(3)}-D_{\alpha \beta \gamma}^{f,(3)}\right]}{\partial t}=-\frac{1}{\tau} \Pi_{\alpha \beta}^{f^{n e q},(n)}+\Pi_{\alpha \beta}^{F,(n)},}{\partial x_{\gamma}}=\rho \mathcal{F}_{\gamma} u_{\gamma}+\rho \dot{q} .
\end{gathered}
$$

We recognize mass, momentum and energy conservation along with an evolution equation for the stress tensor, let us analyze this equation. Using the kinetic tensor equation Eq. (4), the force terms Eqs. $(103,105)$ along with the 2nd and 3rd order macroscopic moments of the equilibrium population lead to the stress tensor equation,

$$
\begin{gathered}
-\Pi_{\alpha \beta}^{f^{n e q},(2)}=\tau \rho c_{s}^{2}\left[\frac{\partial u_{\alpha}}{\partial x_{\beta}}+\frac{\partial u_{\beta}}{\partial x_{\alpha}}-\delta_{\alpha \beta} \frac{2}{3} \frac{\partial u_{\gamma}}{\partial x_{\gamma}}\right] \\
+\tau\left\{\frac{\partial \Pi_{\alpha \beta}^{f^{n e q},(2)}}{\partial t}+\frac{\partial\left[\Pi_{\alpha \beta \gamma}^{f^{n e q},(3)}-D_{\alpha \beta \gamma}^{f^{n e q},(3)}\right]}{\partial x_{\gamma}}\right\}-\tau\left[u_{\alpha} \frac{\partial \Pi_{\beta \gamma}^{f^{n e q},(2)}}{\partial x_{\gamma}}+u_{\beta} \frac{\partial \Pi_{\alpha \gamma}^{f^{n e q},(2)}}{\partial x_{\gamma}}\right],
\end{gathered}
$$

with $\tau \rho c_{s}^{2}=\mu$. This is the exact same evolution equation as in the athermal case. Because equilibrium moments are enslaved to low order macroscopic moments the only place where the higher order moments have an impact in thermo-hydrodynamic equations is through $\Pi_{\alpha \beta \gamma}^{f^{n e q},(3)}$. Fortunately here we used a hybrid recursive regularized collision kernel so that even if higher order macroscopic equations are unphysical their effect on low order macroscopic equations is explicitly filtered during non-equilibrium reconstruction ${ }^{49,65}$. To find out in which cases the stress-tensor evolution equation is sufficiently close to its expected value we use the same nondimensionalization as in the athermal case, except that $Q_{0}=U_{0} \Pi_{0}$ is known because of the RR collision kernel. Also note that $c_{s}$ is not anymore related to the physical sound speed, therefore changing the definition of the Mach number to $M a=U_{0} / \sqrt{\gamma r T_{0}}$. This leads to

$$
\begin{gathered}
-\Pi_{\alpha \beta}^{*, f^{n e q},(2)}=\mathcal{T}_{\alpha \beta}^{*}+\frac{M a^{2}}{\operatorname{Re}} \frac{\gamma r T_{0}}{c_{s}^{2}} \frac{1}{\rho^{*}} \frac{\partial\left[\Pi_{\alpha \beta \gamma}^{*, f^{n e q},(3)}-D_{\alpha \beta \gamma}^{*, f^{n e q},(3)}\right]}{\partial x_{\gamma}^{*}} \\
-\frac{M a^{2}}{\operatorname{Re}} \frac{\gamma r T_{0}}{c_{s}^{2}} \frac{1}{\rho^{*}}\left[u_{\alpha}^{*} \frac{\partial \Pi_{\beta \gamma}^{*, f^{n e q},(2)}}{\partial x_{\gamma}^{*}}+u_{\beta}^{*} \frac{\partial \Pi_{\alpha \gamma}^{*, f^{n e q},(2)}}{\partial x_{\gamma}^{*}}\right]+\frac{\mu}{\rho_{0} c_{s}^{2} t_{s}} \frac{1}{\rho^{*}} \frac{\partial \Pi_{\alpha \beta}^{*, f^{n e q},(2)}}{\partial t^{*}} .
\end{gathered}
$$


Note that defining the CFL number ${ }^{24}$ as

$$
C F L=\frac{U_{0}+\sqrt{\gamma r T_{0}}}{\Delta x / \Delta t},
$$

and reminding that in the acoustic scaling $c_{s}=\Delta x /(\sqrt{3} \Delta t)$ we can see that

$$
\frac{\gamma r T_{0}}{c_{s}^{2}}=\frac{3 C F L^{2}}{(M a+1)^{2}} .
$$

By assuming that our fastest relevant phenomenon is the convection, such that $t_{s}=L_{0} / U_{0}$, we can rearrange Eq. (117) into

$$
-\Pi_{\alpha \beta}^{*, f^{n e q},(2)}=\mathcal{T}_{\alpha \beta}^{*}+O\left(\frac{M a^{2} C F L^{2}}{\operatorname{Re}(M a+1)^{2}}\right),
$$

which is the consistency condition of the pressure-based model ${ }^{7}$. This very simple expression was obtainable only because we used a collision kernel in which the scaling of third order nonequilibrium moments is algebraically enslaved to variables whose scaling is known (see Eq. (95)). This equation shows a very special feature of LBMs: the discretized continuous equations directly depends on the CFL number.

\section{THERMAL RR- $\rho$}

This scheme have been thoroughly used in the literature to perform simulations on compressible ${ }^{31,32}$, combustion $^{17,69}$. Mass and momentum are solved by the LB algorithm while the energy and potential species conservation are solved by a FD scheme, as already presented in the last section.

\section{A. RR- $\rho$ numerical scheme}

Contrarily to the RR-p model this scheme encompasses the thermal effects coming from the FD energy equation inside the 2 nd and 3 rd order equilibrium moments, leading to a more complex equilibrium distribution function.

- Step 1 : Equilibrium construction The equilibrium is expanded as

$$
\begin{aligned}
f_{i}^{e q} & =\omega_{i}\left\{\mathcal{H}^{(0)} \rho+\frac{\mathcal{H}_{i \alpha}^{(1)}}{c_{s}^{2}} \rho u_{\alpha}+\frac{\mathcal{H}_{i \alpha \beta}^{(2)}}{2 c_{s}^{4}}\left[\rho u_{\alpha} u_{\beta}+\rho c_{s}^{2}(\theta-1) \delta_{\alpha \beta}\right]\right. \\
+ & \left.\frac{\mathcal{H}_{i \alpha \beta \gamma}^{(3)}}{6 c_{s}^{6}}\left\{\rho u_{\alpha} u_{\beta} u_{\gamma}+\rho c_{s}^{2}(\theta-1)\left[u_{\gamma} \delta_{\alpha \beta}+u_{\beta} \delta_{\gamma \alpha}+u_{\alpha} \delta_{\beta \gamma}\right]\right\}\right\},
\end{aligned}
$$

where $\theta$ keeps its definition (101). 
- Step 2 : Force construction The forcing population is simply extended to second order,

$$
F_{i} \equiv \omega_{i}\left\{\mathcal{H}^{(0)} a^{F,(0)}+\frac{\mathcal{H}_{i \alpha}^{(1)}}{c_{s}^{2}} a_{\alpha}^{F,(1)}+\frac{\mathcal{H}_{i \alpha \beta}^{(2)}}{2 c_{s}^{4}} a_{\alpha \beta}^{F,(2)}\right\},
$$

with its Hermite moments defined as

$$
\begin{aligned}
a_{\alpha \beta}^{F,(2)} & =-\frac{\partial D_{\alpha \beta \gamma}^{f^{e q},(3)}}{\partial x_{\gamma}}+p\left(\frac{2}{3}-\frac{r}{c_{v}}\right) \frac{\partial u_{\gamma}}{\partial x_{\gamma}} \delta_{\alpha \beta} \\
& +\frac{r \delta_{\alpha \beta}}{c_{v}}\left(\rho \dot{q}+\dot{m} \frac{u_{\gamma}^{2}}{2}\right) \\
& +\rho \mathcal{F}_{\alpha} u_{\beta}+\rho \mathcal{F}_{\beta} u_{\alpha}-\dot{m}\left(u_{\alpha} u_{\beta}+c_{s}^{2} \delta_{\alpha \beta}\right), \\
a_{\alpha}^{F,(1)} & =\rho \mathcal{F}_{\alpha}, \\
a^{F,(0)} & =\dot{m} .
\end{aligned}
$$

- Step 3 : Non-equilibrium construction To allow a fair comparison with the pressurebased model, let us use again the recursive regularized collision operator, Eq. (95).

- Step 4 : Collision Thanks to previous steps $f_{i}^{e q}, F_{i}$ and $\bar{f}_{i}^{\text {neq }}$ have been built, compute the collided population $f_{i}^{c o l}$ such that

$$
f_{i}^{c o l}(t, \boldsymbol{x})=f_{i}^{e q}(t, \boldsymbol{x})+\left(1-\frac{\Delta t}{\bar{\tau}}\right) \bar{f}_{i}^{n e q}(t, \boldsymbol{x})+\frac{\Delta t}{2} F_{i}(t, \boldsymbol{x}) .
$$

- Step 5 : Streaming Shift the populations according to

$$
\bar{f}_{i}(t+\Delta t, \boldsymbol{x})=f_{i}^{c o l}\left(t, \boldsymbol{x}-\boldsymbol{c}_{\boldsymbol{i}} \Delta t\right) .
$$

- Step 6 : Coupling update Here simply solve the explicit numerical scheme Eq. (99) to get $[\rho E](t+\Delta t, \boldsymbol{x})$.

- Step 7 : Update macroscopic Using the macroscopic update rule Eq. (60) for $n=0,1,2$ respectively leads to

$$
\begin{aligned}
& \rho(t+\Delta t, \boldsymbol{x})=\sum_{i}^{q} \bar{f}_{i}(t+\Delta t, \boldsymbol{x})+\frac{\Delta t}{2} \dot{m}(t+\Delta t, \boldsymbol{x}), \\
& \rho u_{\alpha}(t+\Delta t, \boldsymbol{x})=\sum_{i}^{q} c_{i \alpha} \bar{f}_{i}(t+\Delta t, \boldsymbol{x})+\frac{\Delta t}{2}\left[\rho \mathcal{F}_{\alpha}\right](t+\Delta t, \boldsymbol{x}), \\
& \left(1+\frac{\Delta t}{2 \tau}\right) \Pi_{\alpha \beta}^{f^{n e q}(t+\Delta t, \boldsymbol{x}),(2)}=\Pi_{\alpha \beta}^{\bar{f}(t+\Delta t, \boldsymbol{x}),(2)}-\Pi_{\alpha \beta}^{f^{e q}(t+\Delta t, \boldsymbol{x}),(2)}+\frac{\Delta t}{2} \Pi_{\alpha \beta}^{F(t+\Delta t, \boldsymbol{x}),(2)} .
\end{aligned}
$$

Then compute $T(t+\Delta t, \boldsymbol{x})$, from $[\rho E](t+\Delta t, \boldsymbol{x}), u_{\alpha}(t+\Delta t, \boldsymbol{x})$ and $\rho(t+\Delta t, \boldsymbol{x})$. 


\section{B. Continuous equivalent equations}

Now that macroscopic quantities, namely mass, velocity, stress-tensor and temperature, $\rho(t+$ $\Delta t, \boldsymbol{x}), u_{\alpha}(t+\Delta t, \boldsymbol{x}), \Pi_{\alpha \beta}^{f^{n e q},(2)}(t+\Delta t, \boldsymbol{x})$ and $T(t+\Delta t, \boldsymbol{x})$ have been explicitly updated let us analyze the equivalent continuous equations of the system Eqs. $(128,129,130,99)$ and compare it with the target set of equations Eqs. $(1,2,6)$. The Taylor expansion leads to the system of equations

$$
\begin{gathered}
\frac{\partial \rho}{\partial t}+\frac{\partial \rho u_{\beta}}{\partial x_{\beta}}=\dot{m}, \\
\frac{\partial \rho u_{\alpha}}{\partial t}+\frac{\partial\left[\rho u_{\alpha} u_{\beta}+p \delta_{\alpha \beta}+\Pi_{\alpha \beta}^{f^{n e q},(2)}\right]}{\partial x_{\beta}}=\rho \mathcal{F}_{\alpha}, \\
\frac{\partial \Pi_{\alpha \beta}^{f,(2)}+\frac{\partial\left[\Pi_{\alpha \beta \gamma}^{f,(3)}-D_{\alpha \beta \gamma}^{f,(3)}\right]}{\partial t}=-\frac{1}{\tau} \Pi_{\alpha \beta}^{f^{n e q},(n)}+\Pi_{\alpha \beta}^{F,(n)},}{\partial \rho E}+\frac{\partial\left[(\rho E+p) u_{\beta}+q_{\beta}+u_{\alpha} \Pi_{\alpha \beta}^{f^{n e q},(2)}\right]}{\partial x_{\beta}}=\rho \mathcal{F}_{\gamma} u_{\gamma}+\rho \dot{q} .
\end{gathered}
$$

We shall now deduce the stress tensor evolution equation. First, using Eqs. $(131,132)$ to isolate the pressure time derivative $\partial p / \partial t$ inside Eq. (134) and assuming that the adiabatic exponent $\gamma$ is a constant leads to the pressure equation,

$$
\frac{c_{v}}{r}\left(\frac{\partial p}{\partial t}+\frac{\partial p u_{\beta}}{\partial x_{\beta}}\right)+p \frac{\partial u_{\gamma}}{\partial x_{\gamma}}+\frac{\partial q_{\gamma}}{\partial x_{\gamma}}+\Pi_{\beta \gamma}^{f^{n e q},(2)} \frac{\partial u_{\beta}}{\partial x_{\gamma}}=\rho \dot{q}+\dot{m} \frac{u_{\gamma}^{2}}{2}
$$

with $c_{v} / r=1 /(\gamma-1)$. Then injecting Eqs. $(4,135,123-125)$ into Eqs. (133) finally leads to

$$
\begin{aligned}
-\Pi_{\alpha \beta}^{f^{n e q},(2)} & =\tau p\left[\frac{\partial u_{\alpha}}{\partial x_{\beta}}+\frac{\partial u_{\beta}}{\partial x_{\alpha}}-\delta_{\alpha \beta} \frac{2}{3} \frac{\partial u_{\gamma}}{\partial x_{\gamma}}\right]+\tau\left\{\frac{\partial \Pi_{\alpha \beta}^{f^{n e q},(2)}}{\partial t}+\frac{\partial\left[\Pi_{\alpha \beta \gamma}^{f^{n e q},(3)}-D_{\alpha \beta \gamma}^{f^{n e q},(3)}\right]}{\partial x_{\gamma}}\right\} \\
- & \tau\left[u_{\alpha} \frac{\partial \Pi_{\beta \gamma}^{f^{n e q},(2)}}{\partial x_{\gamma}}+u_{\beta} \frac{\partial \Pi_{\alpha \gamma}^{f^{n e q},(2)}}{\partial x_{\gamma}}\right]+\tau \frac{r \delta_{\alpha \beta}}{c_{v}}\left[-\frac{\partial q_{\gamma}}{\partial x_{\gamma}}-\Pi_{\beta \gamma}^{f^{n e q},(2)} \frac{\partial u_{\beta}}{\partial x_{\gamma}}\right]
\end{aligned}
$$

with $\tau p=\mu$. Here we end up with a slightly different evolution equation for the stress tensor when compared to the pressure-based and athermal cases. Again the higher order unphysical moments are filtered out because $\Pi_{\alpha \beta \gamma}^{f^{n e q},(3)}$ was reconstructed following Eq. (95).

To allow a fair comparison with the RR-p model we use the previously defined nondimensionalization. This leads to

$$
\begin{aligned}
& -\Pi_{\alpha \beta}^{*, f^{n e q},(2)}=\Pi_{\alpha \beta}^{*, N S}+\frac{\gamma M a^{2}}{\operatorname{Re}} \frac{1}{\rho^{*} T^{*}}\left\{\frac{\partial\left[\Pi_{\alpha \beta \gamma}^{*, f^{n e q},(3)}-D_{\alpha \beta \gamma}^{*, f^{n e q},(3)}\right]}{\partial x_{\gamma}^{*}}-u_{\alpha}^{*} \frac{\partial \Pi_{\beta \gamma}^{*, f^{n e q},(2)}}{\partial x_{\gamma}^{*}}-u_{\beta}^{*} \frac{\partial \Pi_{\alpha \gamma}^{*, f^{n e q},(2)}}{\partial x_{\gamma}^{*}}\right\} \\
& +\frac{\mu}{\rho_{0} R T_{0} t_{s}} \frac{1}{\rho^{*} T^{*}} \frac{\partial \Pi_{\alpha \beta}^{*, f^{n e q},(2)}}{\partial t^{*}}+\frac{\gamma}{\operatorname{PrRe}} \frac{\delta_{\alpha \beta}}{\rho^{*} T^{*}}\left[\frac{\partial^{2} T^{*}}{\partial x_{\gamma}^{*} \partial x_{\gamma}^{*}}\right]-\frac{\gamma M a^{2}(\gamma-1)}{\operatorname{Re}} \frac{\delta_{\alpha \beta}}{\rho^{*} T^{*}}\left[\Pi_{\beta \gamma}^{*, f^{n e q},(2)} \frac{\partial u_{\beta}^{*}}{\partial x_{\gamma}^{*}}\right] .(137)
\end{aligned}
$$


Assuming that convection is dominant, $t_{s}=L_{0} / U_{0}$, Eq. (137) can be recast into

$$
-\Pi_{\alpha \beta}^{*, f^{n e q},(2)}=\Pi_{\alpha \beta}^{*, N S}+O\left(\frac{M a^{2}}{R e}\right)+O\left(\frac{1}{R e P r}\right) .
$$

This last equation should be compared to Eq. (120). While the consistency defect of the densitybased model increase with $M a$ it remains bounded for the pressure-based. Another interesting difference between density-based on one side and both athermal and pressure-based on the other side is that the former model does not have a decreasing consistency defect when the CFL is decreased, an evident limitation for simulating compressible flows.

\section{ILLUSTRATION: SPHERICAL FLAME}

In order to illustrate that different consistency defects can lead to very different results a circular laminar flame is simulated with both the density-based (Sec. VIII) and the pressure-based (Sec. VII) models, as adapted for reactive flows ${ }^{17,18}$. This test case was found to particularly aggravate spurious currents effects because of the presence of heat-release along a curved density gradient. This two dimensional test is setup as follows: the domain size is chosen as $4 \mathrm{~cm} \times 4 \mathrm{~cm}$, with a grid spacing $\Delta x=0.2 \mathrm{~mm}$. The domain is initialized with fresh stoichiometric mixture of propane and air at $300 \mathrm{~K}$ and $1 \mathrm{~atm}$ and a spot of corresponding burnt gases in its center. The thermodynamic, transport and chemical closures applied in the current work are identical to those in $^{17}$, which correspond to a laminar flame speed $S_{L} \approx 0.25 \mathrm{~m} / \mathrm{s}$ and a thermal flame thickness $\delta_{L} \approx 0.6 \mathrm{~mm}$. It is worth noting that the grid is quite coarse, corresponding to around three points in one thermal flame thickness. The boundary conditions are set to characteristic in/out boundaries with a far-field pressure $p_{\infty}=1 \mathrm{~atm}$.

The flow fields of two very early time steps of simulation are presented in Fig. 1. It can be seen that the density-based scheme creates spurious currents near the flame front which leads to unstable simulation, whereas the pressure-based scheme presents the expected behavior.

The temperature, heat release rate (HR) and velocity fields from the simulation pressure-based are given at a later instant in Fig. 2. Profiles are seen to remain circular even when approaching the boundary, showing both the isotropy of the scheme as well as the effectiveness of the characteristic boundary.

This comparison between density-based and pressure-based formulations illustrates how spurious currents can be triggered by defects of consistency of the stress-tensor. 

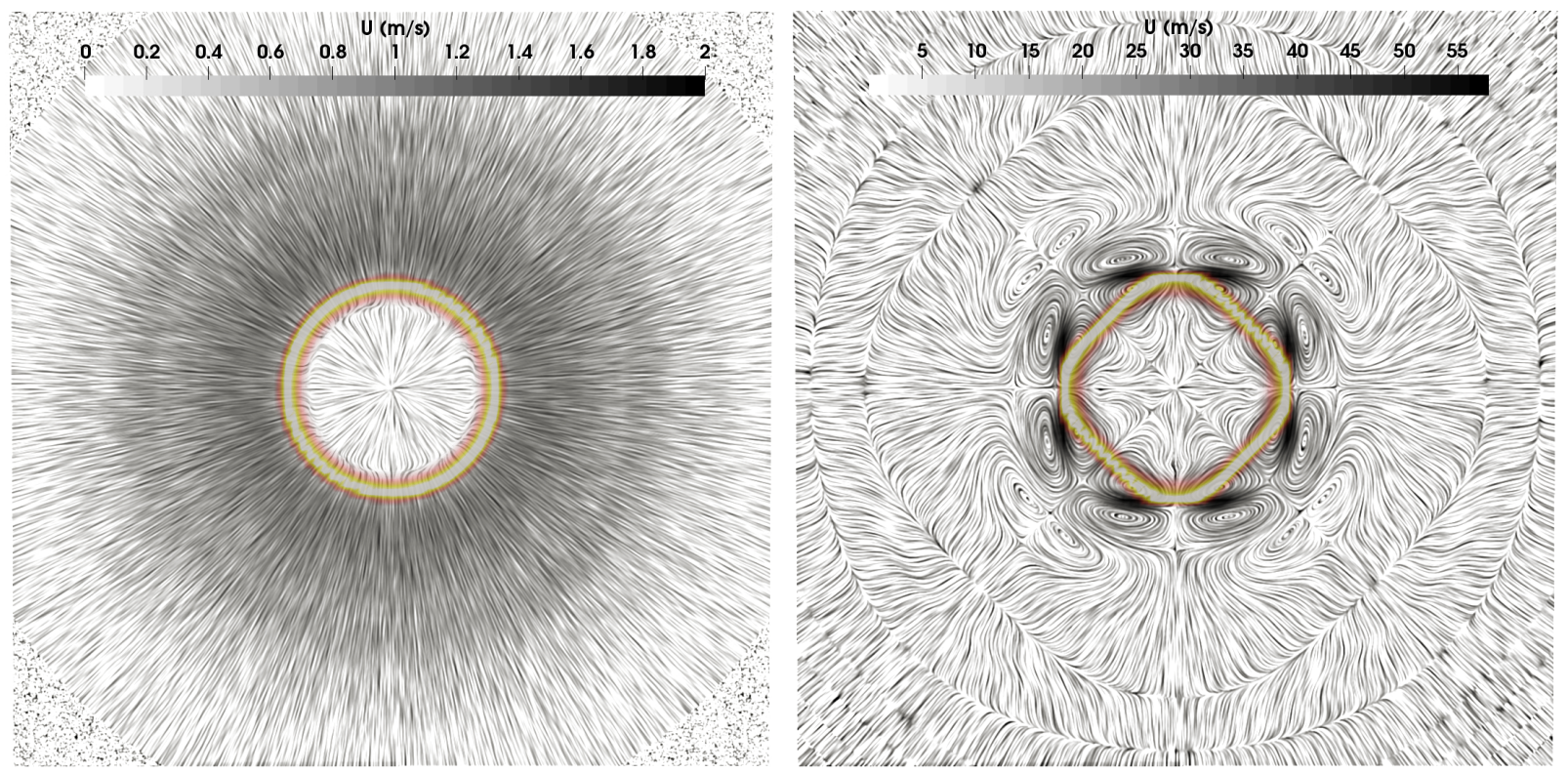

(a) $\mathrm{t}=2.0 \times 10^{-2} \mathrm{~ms}$
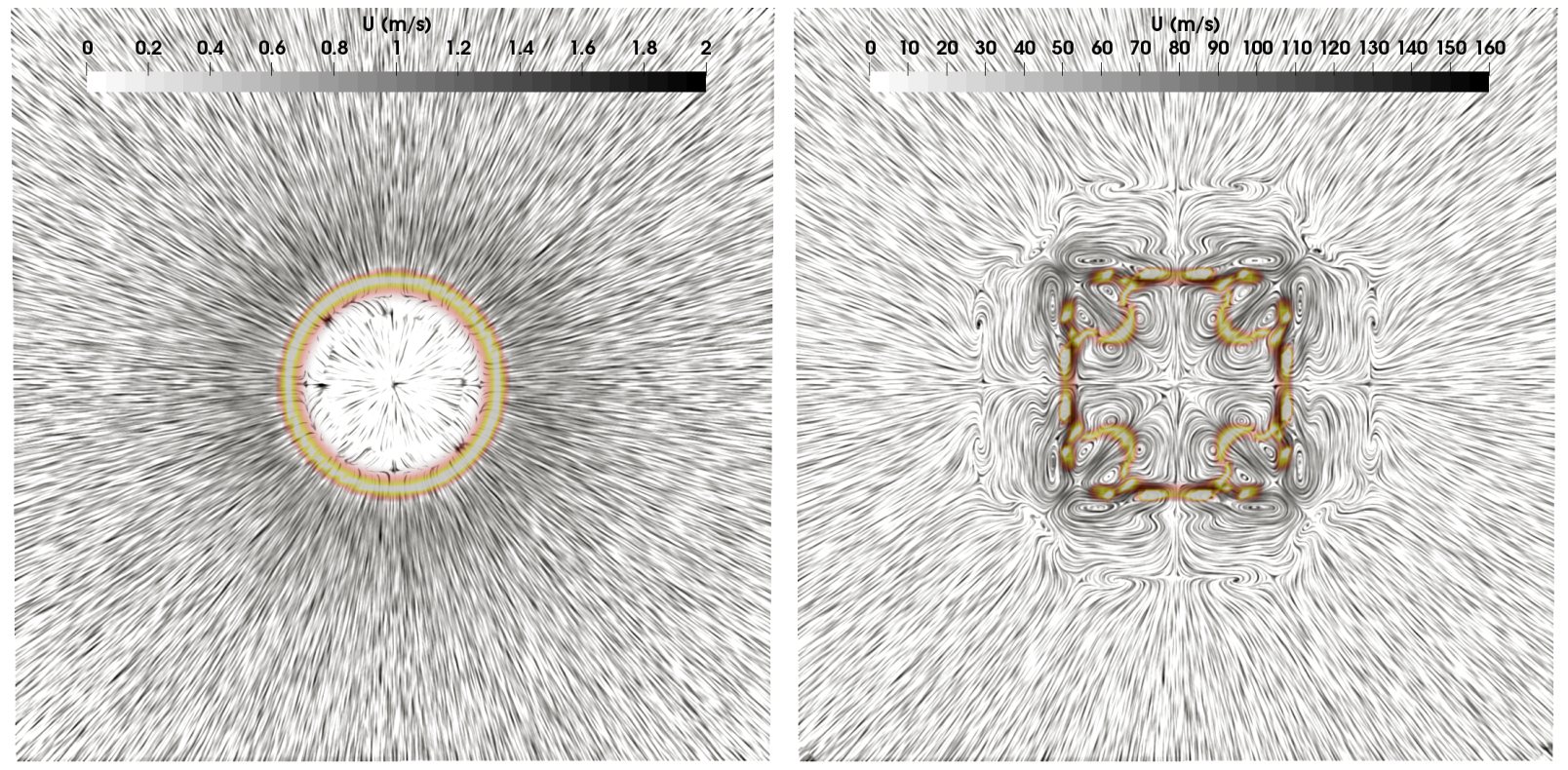

(b) $\mathrm{t}=4.0 \times 10^{-2} \mathrm{~ms}$

Figure 1: Streamlines of the 2D circular flame simulation colored by velocity magnitude (in $\mathrm{m} / \mathrm{s}$ ). Left column: pressure-based model ${ }^{18}$, right column: density-based model ${ }^{17}$. Note the very different ranges of velocity magnitude from the two methods. The yellow contour is the heat release rate peak indicating the flame front. 

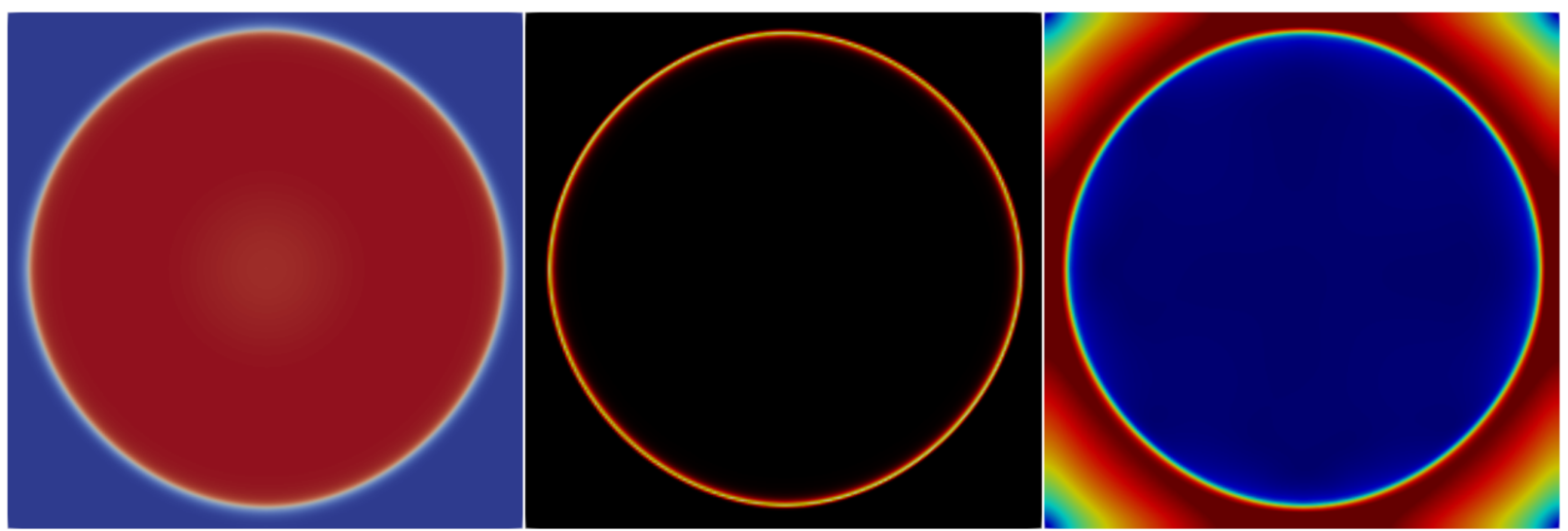

Figure 2: Visualization of the temperature, heat release rate and velocity magnitude fields of the $2 \mathrm{D}$ circular flame as obtained with the pressure-based formulation ${ }^{18}$, at $t=9.24 \mathrm{~s}$.

\section{CONCLUSION}

We have presented a new systematic method to analyze Lattice-Boltzmann models. Based on a Taylor-Expansion, it allows to derive the set of macroscopic equations consistent with the model. The analysis only requires the assumption of a sufficiently small time discretization $\Delta t \rightarrow 0$, as is usual for consistency studies.

A major advantage of the method over the traditional Chapman-Enskog framework is that it allows to study more carefully numerical errors. In light of this Taylor-Expansion, collision kernel's effects on macroscopic variables are reinterpreted as a closure for the stress-tensor evolution equation. Numerical coupling of Lattice-Boltzmann models with other numerical schemes are shown to create error terms whose scalings are more complicated than what the ChapmanEnskog expansion suggests. Apart the usual low-Knudsen assumption it is found that Mach, Prandtl and CFL numbers can also intervene in consistency conditions of Lattice-Boltzmann models. Note that although we focused on standard, nearest-neighbors lattices such as D2Q9, D3Q19 and D3Q27, the presented method can be extended to larger lattices.

Consistency errors are analyzed for three models: (i) athermal model, (ii) a density-based compressible model, (iii) a pressure-based compressible model. The consistency errors reported in Tab. V show that, for the athermal model (i), the consistency error decreases with the CFL and increases with the Mach number. For compressible models, two distinct behaviors were found:

- increasing consistency error with increasing Mach number, and bounded error with decreasing CFL number, for the density-based model (ii), 
Table V: Summary of consistency errors for the stress tensor, for the three models studied. Here $\widetilde{M} a$ is the lattice-speed based Mach number, Eq. (87)

\begin{tabular}{lll}
\hline \hline Model & Stress tensor error & Equation \\
\hline Athermal model (Section V) & $O\left(\frac{\widetilde{M a}^{2}}{R e}\right)$ & $(90)$ \\
Compressible pressure-based (Section VII) & $O\left(\frac{M a^{2} C F L^{2}}{R e(M a+1)^{2}}\right)$ & (120) \\
Compressible density-based (Section VIII) & $O\left(\frac{M a^{2}}{R e}\right)+O\left(\frac{1}{R e P r}\right)$ & (138) \\
\hline \hline
\end{tabular}

- bounded consistency error with increasing Mach number and vanishing error with decreasing CFL number, for the pressure-based model (iii).

The Taylor analysis presented here therefore clearly favors the pressure-based model, while the Chapman-Enskog expansion was unable to discriminate the two models.

\section{ACKNOWLEDGEMENTS}

We thank Gauthier Wissocq and Pierre Sagaut for inspiring discussions. We also acknowledge support from Labex MEC (ANR-10-LABX-0092), the A*MIDEX project (ANR-11-IDEX-0001-02), funded by the "Investissements d'Avenir" and the French Space agency (CNES) for supporting Song Zhao at M2P2.

\section{DATA AVAILABILITY}

The data that support the findings of this study are available from the corresponding author upon reasonable request.

\section{DECLARATION OF INTERESTS}

The authors report no conflict of interest. 


\section{Appendix A: Second order of accuracy of LBMs}

To study the order of accuracy let us use Eq. (63) evaluated for the $(n+1)$-order moment along with Eq. (59) to get

$$
\Delta t \frac{\partial \Pi_{\alpha_{1} \ldots \alpha_{n+2}}^{f^{c o l}(t, x),(n+2)}}{\partial x_{\alpha_{n+2}}}=\Pi_{\alpha_{1} \ldots \alpha_{n+1}}^{f^{c o l}(t, x),(n+1)}-\prod_{\alpha_{1} \ldots \alpha_{n+1}}^{\bar{f}(t+\Delta t, \boldsymbol{x}),(n+1)}+O\left(\Delta t^{2}\right),
$$

which can be injected back into Eq. (63), then using Eq. (59) leads to

$$
\Pi_{\alpha_{1} \ldots \alpha_{n}}^{\bar{f}(t+\Delta t, \boldsymbol{x}),(n)}-\Pi_{\alpha_{1} \ldots \alpha_{n}}^{f^{c o l}(t, \boldsymbol{x}),(n)}=-\frac{\Delta t}{2} \frac{\partial}{\partial x_{\alpha_{n+1}}}\left[\Pi_{\alpha_{1} \ldots \alpha_{n+1}}^{f^{c o l}(t, \boldsymbol{x}),(n+1)}+\Pi_{\alpha_{1} \ldots \alpha_{n+1}}^{\bar{f}(t+\Delta t, \boldsymbol{x}),(n+1)}\right]+O\left(\Delta t^{3}\right) .
$$

On the other hand, Eqs. $(52,55,50)$ leads to

$$
\begin{aligned}
\Pi_{\alpha_{1} \ldots \alpha_{n}}^{f c o l}(t, \boldsymbol{x}),(n) & =\left[\Pi_{\alpha_{1} \ldots \alpha_{n}}^{f,(n)}-\frac{\Delta t}{2}\left(\frac{1}{\tau} \Pi_{\alpha_{1} \ldots \alpha_{n}}^{f f e q}-\Pi_{\alpha_{1} \ldots \alpha_{n}}^{f(n)}\right)\right](t, \boldsymbol{x}), \\
\Pi_{\alpha_{1} \ldots \alpha_{n}}^{\bar{f}(t+\Delta t, \boldsymbol{x}),(n)} & =\left[\Pi_{\alpha_{1} \ldots \alpha_{n}}^{f,(n)}+\frac{\Delta t}{2}\left(\frac{1}{\tau} \Pi_{\alpha_{1} \ldots \alpha_{n}}^{f n e q}-\Pi_{\alpha_{1} \ldots \alpha_{n}}^{f,(n)}\right)\right](t+\Delta t, \boldsymbol{x}) .
\end{aligned}
$$

At this point it is curious to note that the collision forcing $\tau^{-1} \Pi_{\alpha_{1} \ldots \alpha_{n}}^{f n e q},(n)$ and the external forcing $\Pi_{\alpha_{1} \ldots \alpha_{n}}^{F,(n)}$ have the exact same treatment in the algorithm. Therefore the collision discretization is nothing else than a particular Guo forcing applied to a collisionless discretized BE. Also note that

$$
\Pi_{\alpha_{1} \ldots \alpha_{n}}^{f c o l}(t, \boldsymbol{x}),(n)+\Pi_{\alpha_{1} \ldots \alpha_{n}}^{\bar{f}(t+\Delta t, \boldsymbol{x}),(n)} \approx \Pi_{\alpha_{1} \ldots \alpha_{n}}^{f(t, \boldsymbol{x}),(n)}+\Pi_{\alpha_{1} \ldots \alpha_{n}}^{f(t+\Delta t, \boldsymbol{x}),(n)}+O\left(\Delta t^{2}\right) .
$$

Injecting Eq. (A5) and Eq. (A4) respectively in the right hand side and left hand side of Eq. (A2) leads to the general second order numerical scheme :

$$
\begin{array}{r}
\Pi_{\alpha_{1} \ldots \alpha_{n}}^{f(t, \Delta t,(n)}-\Pi_{\alpha_{1} \ldots \alpha_{n}}^{f(t, x),(n)}=-\frac{\Delta t}{2} \frac{\partial}{\partial x_{\alpha_{n+1}}}\left[\Pi_{\alpha_{1} \ldots \alpha_{n+1}}^{f,(n+1)}(t, \boldsymbol{x})+\Pi_{\alpha_{1} \ldots \alpha_{n+1}}^{f,(n+1)}(t+\Delta t, \boldsymbol{x})\right] \\
-\frac{\Delta t}{2}\left[\left(\frac{1}{\tau} \prod_{\alpha_{1} \ldots \alpha_{n}}^{f^{n e q},(n)}-\Pi_{\alpha_{1} \ldots \alpha_{n}}^{F,(n)}\right)(t, \boldsymbol{x})+\left(\frac{1}{\tau} \prod_{\alpha_{1} \ldots \alpha_{n}}^{f n e q(n)}-\Pi_{\alpha_{1} \ldots \alpha_{n}}^{F,(n)}\right)(t+\Delta t, \boldsymbol{x})\right]+O\left(\Delta t^{3}\right),
\end{array}
$$

or equivalently

$$
\begin{aligned}
\frac{\prod_{\alpha_{1} \ldots \alpha_{n}}^{f(t+\Delta t, \boldsymbol{x}),(n)}-\Pi_{\alpha_{1} \ldots \alpha_{n}}^{f(t, x),(n)}}{\Delta t} & =\frac{1}{2}\left\{\left[-\frac{\partial \Pi_{\alpha_{1} \ldots \alpha_{n+1}}^{f,(n+1)}}{\partial x_{\alpha_{n+1}}}-\frac{1}{\tau} \Pi_{\alpha_{1} \ldots \alpha_{n}}^{f n e q}+\Pi_{\alpha_{1} \ldots \alpha_{n}}^{f,(n)}\right](t+\Delta t, \boldsymbol{x})\right. \\
+ & {\left.\left[-\frac{\partial \Pi_{\alpha_{1} \ldots \alpha_{n+1}}^{f,(n+1)}}{\partial x_{\alpha_{n+1}}}-\frac{1}{\tau} \Pi_{\alpha_{1} \ldots \alpha_{n}}^{f n e q}+\Pi_{\alpha_{1} \ldots \alpha_{n}}^{f,(n)}\right](t, \boldsymbol{x})\right\}+O\left(\Delta t^{2}\right) . }
\end{aligned}
$$

As already highlighted in the literature on the $f$ equation itself $^{70}$ we recognize in this last equation a second order accurate Crank-Nicolson scheme whose limit $\Delta t \rightarrow 0$ is Eq. (64). Note that 
rigorously speaking this 2nd order accuracy stands for each of the $q$ moments associated to the DdQq lattice only in the BGK case. In other words Eq. (A4) is strictly verified only for BGK kernel. Meaning that depending on the chosen collision kernel the schemes associated to higher order moments than $\Pi^{(0)}$ and $\Pi_{\alpha}^{(1)}$ may not exhibit a 2nd order accuracy ${ }^{65}$.

\section{REFERENCES}

${ }^{1}$ D. Hilbert, "Mathematical problems," Bulletin of the American Mathematical Society 8, 437-479 (1902).

${ }^{2}$ L. Boltzmann, "Weitere studien über das wärmegleichgewicht unter gasmolekülen," in Kinetische Theorie II (Springer, 1970) pp. 115-225.

${ }^{3}$ C. Cercignani, "The boltzmann equation," in The Boltzmann equation and its applications (Springer, 1988) pp. 40-103.

${ }^{4}$ L. Saint-Raymond, Hydrodynamic limits of the Boltzmann equation, 1971 (Springer Science \& Business Media, 2009).

${ }^{5}$ A. Gorban and I. Karlin, "Hilbert's 6th problem: exact and approximate hydrodynamic manifolds for kinetic equations," Bulletin of the American Mathematical Society 51, 187-246 (2014).

${ }^{6}$ C. Villani, "Limites hydrodynamiques de l'équation de boltzmann," Séminaire Bourbaki 2000, 365-405 (2001).

${ }^{7}$ G. Farag, S. Zhao, T. Coratger, P. Boivin, G. Chiavassa, and P. Sagaut, "A pressure-based regularized lattice-boltzmann method for the simulation of compressible flows," Physics of Fluids 32, $066106(2020)$.

${ }^{8}$ J. Latt, C. Coreixas, J. Beny, and A. Parmigiani, "Efficient supersonic flow simulations using lattice boltzmann methods based on numerical equilibria," Philosophical Transactions of the Royal Society A 378, 20190559 (2020).

${ }^{9}$ S. Guo, Y. Feng, J. Jacob, F. Renard, and P. Sagaut, “An efficient lattice boltzmann method for compressible aerodynamics on d3q19 lattice," Journal of Computational Physics , 109570 (2020).

${ }^{10}$ M. H. Saadat and I. V. Karlin, “Arbitrary lagrangian-eulerian formulation of lattice boltzmann model for compressible flows on unstructured moving meshes,” Physics of Fluids 32, 046105 (2020).

${ }^{11} \mathrm{R}$. Zhang, X. He, and S. Chen, "Interface and surface tension in incompressible lattice boltzmann multiphase model," Computer Physics Communications 129, 121-130 (2000). 
${ }^{12}$ T. Lee and C.-L. Lin, "A stable discretization of the lattice boltzmann equation for simulation of incompressible two-phase flows at high density ratio," Journal of Computational Physics 206, $16-47$ (2005).

${ }^{13} \mathrm{X}$. He, S. Chen, and R. Zhang, "A lattice boltzmann scheme for incompressible multiphase flow and its application in simulation of rayleigh-taylor instability," Journal of computational physics 152, 642-663 (1999).

${ }^{14}$ R. Zhang, X. He, G. Doolen, and S. Chen, "Surface tension effects on two-dimensional twophase kelvin-helmholtz instabilities," Advances in water resources 24, 461-478 (2001).

${ }^{15}$ X. He, R. Zhang, S. Chen, and G. D. Doolen, "On the three-dimensional rayleigh-taylor instability," Physics of Fluids 11, 1143-1152 (1999).

${ }^{16}$ T. Inamuro, T. Ogata, S. Tajima, and N. Konishi, "A lattice boltzmann method for incompressible two-phase flows with large density differences," Journal of Computational physics 198, 628-644 (2004).

${ }^{17}$ M. Tayyab, S. Zhao, Y. Feng, and P. Boivin, "Hybrid regularized lattice-boltzmann modelling of premixed and non-premixed combustion processes," Combustion and Flame 211, 173-184 (2020).

${ }^{18}$ M. Tayyab, B. Radisson, C. Almarcha, B. Denet, and P. Boivin, "Experimental and numerical lattice-boltzmann investigation of the darrieus-landau instability," Combustion and Flame 221, 103-109 (2020).

${ }^{19}$ S. A. Hosseini, A. Eshghinejadfard, N. Darabiha, and D. Thévenin, "Weakly compressible lattice boltzmann simulations of reacting flows with detailed thermo-chemical models," Computers \& Mathematics with Applications 79, 141-158 (2020).

${ }^{20}$ S. Chapman and T. Cowling, "The mathematical theory of non-uniform gases, 3rd edn. cambridge math," (1970).

${ }^{21} \mathrm{~J}$. Jacob, O. Malaspinas, and P. Sagaut, “A new hybrid recursive regularised bhatnagar-grosskrook collision model for lattice boltzmann method-based large eddy simulation,” Journal of Turbulence 19, 1051-1076 (2018).

${ }^{22}$ P. L. Bhatnagar, E. P. Gross, and M. Krook, "A model for collision processes in gases. i. small amplitude processes in charged and neutral one-component systems," Physical review 94, 511 (1954).

${ }^{23}$ R. Warming and B. Hyett, "The modified equation approach to the stability and accuracy analysis of finite-difference methods," Journal of computational physics 14, 159-179 (1974). 
${ }^{24}$ R. J. LeVeque, Finite difference methods for ordinary and partial differential equations: steady-state and time-dependent problems (SIAM, 2007).

${ }^{25} \mathrm{E}$. F. Toro, Riemann solvers and numerical methods for fluid dynamics: a practical introduction (Springer Science \& Business Media, 2013).

${ }^{26}$ F. Dubois, "Equivalent partial differential equations of a lattice boltzmann scheme," Computers \& Mathematics with Applications 55, 1441-1449 (2008).

${ }^{27}$ M. Junk, “A finite difference interpretation of the lattice boltzmann method," Numerical Methods for Partial Differential Equations: An International Journal 17, 383-402 (2001).

${ }^{28}$ M. Junk and W.-A. Yong, "Rigorous navier-stokes limit of the lattice boltzmann equation," Asymptotic Analysis 35, 165-185 (2003).

${ }^{29}$ H. Grad, "On the kinetic theory of rarefied gases," Communications on pure and applied mathematics 2, 331-407 (1949).

${ }^{30}$ T. Krüger, H. Kusumaatmaja, A. Kuzmin, O. Shardt, G. Silva, and E. M. Viggen, "The lattice boltzmann method," Springer International Publishing 10, 978-3 (2017).

${ }^{31}$ Y. Feng, P. Boivin, J. Jacob, and P. Sagaut, "Hybrid recursive regularized thermal lattice boltzmann model for high subsonic compressible flows," Journal of Computational Physics 394, 82-99 (2019).

${ }^{32}$ F. Renard, Y. Feng, J. Boussuge, and P. Sagaut, "Improved compressible hybrid lattice boltzmann method on standard lattice for subsonic and supersonic flows," arXiv preprint arXiv:2002.03644 (2020).

${ }^{33}$ Y. Feng, S. Guo, J. Jacob, and P. Sagaut, "Solid wall and open boundary conditions in hybrid recursive regularized lattice boltzmann method for compressible flows," Physics of Fluids 31, 126103 (2019).

${ }^{34}$ H. W. Liepmann and A. Roshko, Elements of gasdynamics (Courier Corporation, 2001).

${ }^{35}$ J. C. Maxwell, "V. illustrations of the dynamical theory of gases.-part i. on the motions and collisions of perfectly elastic spheres," The London, Edinburgh, and Dublin Philosophical Magazine and Journal of Science 19, 19-32 (1860).

${ }^{36}$ C. Cercignani, Rarefied gas dynamics: from basic concepts to actual calculations, Vol. 21 (Cambridge University Press, 2000).

${ }^{37}$ D. Hilbert, "Begründung der kinetischen gastheorie," Mathematische Annalen 72, 562-577 (1912). 
${ }^{38}$ D. Hilbert, “Grundzüge einer allgemeinen theorie der linearen integralgleichungen," in Integralgleichungen und Gleichungen mit unendlich vielen Unbekannten (Springer, 1989) pp. 8-171.

${ }^{39} \mathrm{~S}$. Succi, The lattice Boltzmann equation: for complex states of flowing matter (Oxford University Press, 2018).

${ }^{40}$ G. E. Uhlenbeck, G. W. Ford, G. W. Ford, and E. W. Montroll, Lectures in statistical mechanics, Vol. 1 (Proquest/Csa Journal Division, 1963).

${ }^{41}$ H. Grad, "Note on n-dimensional hermite polynomials," Communications on Pure and Applied Mathematics 2, 325-330 (1949).

${ }^{42}$ X. Shan, X.-F. Yuan, and H. Chen, "Kinetic theory representation of hydrodynamics: a way beyond the navier-stokes equation,” Journal of Fluid Mechanics 550, 413-441 (2006).

${ }^{43}$ J. K. Hunter, "Asymptotic analysis and singular perturbation theory," Department of Mathematics, University of California at Davis , 1-3 (2004).

${ }^{44} \mathrm{H}$. Grad, "Asymptotic theory of the boltzmann equation," The physics of Fluids 6, 147-181 (1963).

${ }^{45}$ H. C. Öttinger, Beyond equilibrium thermodynamics (John Wiley \& Sons, 2005).

${ }^{46} \mathrm{H}$. Struchtrup, “Macroscopic transport equations for rarefied gas flows," in Macroscopic Transport Equations for Rarefied Gas Flows (Springer, 2005) pp. 145-160.

${ }^{47}$ A. Bobylev, "The chapman-enskog and grad methods for solving the boltzmann equation," DoSSR 262, 71-75 (1982).

${ }^{48}$ A. Bobylev, "Instabilities in the chapman-enskog expansion and hyperbolic burnett equations," Journal of statistical physics 124, 371-399 (2006).

${ }^{49}$ C. Coreixas, G. Wissocq, G. Puigt, J.-F. Boussuge, and P. Sagaut, "Recursive regularization step for high-order lattice boltzmann methods," Physical Review E 96, 033306 (2017).

${ }^{50} \mathrm{I}$. Ginzburg, "Consistent lattice boltzmann schemes for the brinkman model of porous flow and infinite chapman-enskog expansion,” Physical Review E 77, 066704 (2008).

${ }^{51}$ N. Frapolli, S. S. Chikatamarla, and I. V. Karlin, "Entropic lattice boltzmann model for compressible flows," Physical Review E 92, 061301 (2015).

${ }^{52}$ N. Frapolli, S. S. Chikatamarla, and I. V. Karlin, "Entropic lattice boltzmann model for gas dynamics: Theory, boundary conditions, and implementation,” Physical Review E 93, 063302 (2016).

${ }^{53}$ N. Frapolli, S. S. Chikatamarla, and I. V. Karlin, "Lattice kinetic theory in a comoving galilean reference frame," Physical review letters 117, 010604 (2016). 
${ }^{54}$ A. Mohamad, Lattice Boltzmann Method, Vol. 70 (Springer, 2011).

${ }^{55}$ Z. Guo and C. Shu, Lattice Boltzmann method and its applications in engineering, Vol. 3 (World Scientific, 2013).

${ }^{56}$ Z. Guo, C. Zheng, and B. Shi, "Discrete lattice effects on the forcing term in the lattice boltzmann method," Physical review E 65, 046308 (2002).

${ }^{57}$ R. Löhner, “Towards overcoming the les crisis," International Journal of Computational Fluid Dynamics 33, 87-97 (2019).

${ }^{58}$ S. Marié, D. Ricot, and P. Sagaut, "Comparison between lattice boltzmann method and navierstokes high order schemes for computational aeroacoustics," Journal of Computational Physics 228, 1056-1070 (2009).

${ }^{59}$ P. Lallemand and L.-S. Luo, "Theory of the lattice boltzmann method: Acoustic and thermal properties in two and three dimensions," Physical review E 68, 036706 (2003).

${ }^{60}$ G. Di Ilio, B. Dorschner, G. Bella, S. Succi, and I. V. Karlin, "Simulation of turbulent flows with the entropic multirelaxation time lattice boltzmann method on body-fitted meshes," Journal of Fluid Mechanics 849, 35-56 (2018).

${ }^{61}$ J. Onishi, Y. Chen, and H. Ohashi, “A lattice boltzmann model for polymeric liquids,” Progress in Computational Fluid Dynamics, an International Journal 5, 75-84 (2005).

${ }^{62}$ F. Osmanlic and C. Körner, "Lattice boltzmann method for oldroyd-b fluids," Computers \& Fluids 124, 190-196 (2016).

${ }^{63}$ V. Küng, F. Osmanlic, M. Markl, and C. Körner, "Comparison of passive scalar transport models coupled with the lattice boltzmann method," Computers \& Mathematics with Applications 79, $55-65(2020)$.

${ }^{64}$ F. Renard, G. Wissocq, J.-F. Boussuge, and P. Sagaut, “A linear stability analysis of compressible hybrid lattice boltzmann methods," arXiv preprint arXiv:2006.08477 (2020).

${ }^{65} \mathrm{G}$. Wissocq, C. Coreixas, and J.-F. Boussuge, "Linear stability of athermal regularized lattice boltzmann methods," arXiv preprint arXiv:2006.07353 (2020).

${ }^{66}$ P.-A. Masset and G. Wissocq, "Linear hydrodynamics and stability of the discrete velocity boltzmann equations," Journal of Fluid Mechanics 897 (2020).

${ }^{67}$ J. Latt and B. Chopard, "Lattice boltzmann method with regularized pre-collision distribution functions," Mathematics and Computers in Simulation 72, 165-168 (2006).

${ }^{68}$ K. K. Mattila, P. C. Philippi, and L. A. Hegele Jr, "High-order regularization in lattice-boltzmann equations," Physics of Fluids 29, 046103 (2017). 
${ }^{69}$ Y. Feng, M. Tayyab, and P. Boivin, "A lattice-boltzmann model for low-mach reactive flows," Combustion and Flame 196, 249 - 254 (2018).

${ }^{70} \mathrm{P}$. J. Dellar, "An interpretation and derivation of the lattice boltzmann method using strang splitting," Computers \& Mathematics with Applications 65, 129-141 (2013). 